Near-Edge X-ray Absorption Fine Structure Spectroscopy of Diamondoid Thiol Monolayers on Gold

T. M. Willey, J. Fabbri, J. R. I. Lee, P. Schreiner, A. A. Fokin, B. A. Tkachenko, N. A. Fokina, J. Dahl, B. Carlson, A. L. Vance, W. Yang, L. J. Terminello, T. van Buuren, N. Melosh

November 27, 2007

Journal of the American Chemical Society 
This document was prepared as an account of work sponsored by an agency of the United States government. Neither the United States government nor Lawrence Livermore National Security, LLC, nor any of their employees makes any warranty, expressed or implied, or assumes any legal liability or responsibility for the accuracy, completeness, or usefulness of any information, apparatus, product, or process disclosed, or represents that its use would not infringe privately owned rights. Reference herein to any specific commercial product, process, or service by trade name, trademark, manufacturer, or otherwise does not necessarily constitute or imply its endorsement, recommendation, or favoring by the United States government or Lawrence Livermore National Security, LLC. The views and opinions of authors expressed herein do not necessarily state or reflect those of the United States government or Lawrence Livermore National Security, LLC, and shall not be used for advertising or product endorsement purposes. 


\title{
Near-Edge X-ray Absorption Fine Structure Spectroscopy of Diamondoid Thiol Monolayers on Gold
}

\author{
Trevor M. Willey ${ }^{*}$, Jason D. Fabbri ${ }^{2}$, Jonathan R. I. Lee ${ }^{1}$, Peter R. Schreiner ${ }^{3}$, \\ Andrey A. Fokin ${ }^{3}$, Boryslav A. Tkachenko ${ }^{3}$, Nataliya A. Fokina ${ }^{3}$, Jeremy E. P. Dahl ${ }^{4}$, \\ Robert M. K. Carlson ${ }^{4}$, Andrew L. Vance ${ }^{5}$, Wanli Yang ${ }^{6}$, Louis J. Terminello ${ }^{1}$, \\ Tony van Buuren ${ }^{1}$, and Nicolas A. Melosh ${ }^{2}$
}

1) Materials Science and Technology Division, Lawrence Livermore National Laboratory, 7000 East Avenue, Livermore CA 94550

2) Materials Science and Engineering, Stanford University, 476 Lomita Mall, Stanford, CA 94305

3) Institute of Organic Chemistry, Justus-Liebig University Giessen, Heinrich-Buff-Ring 58, 35392 Giessen, Germany

4) MolecularDiamond Technologies, Chevron Technology Ventures, 100 Chevron Way, Richmond, CA 94802

5) Materials Chemistry Department, Sandia National Laboratories, 7011 East Avenue, Livermore, CA 94550

6) Advanced Light Source, Lawrence Berkeley National Laboratory, 1 Cyclotron Road, Berkeley, CA 94720

* e-mail address: willey1@1lnl.gov

Keywords:

Self-assembled monolayers, diamondoids, thiols, molecular electronics, nanodiamond, NEXAFS, XPS.

UCRL-JRNL-236817 


\begin{abstract}
Diamondoids, hydrocarbon molecules with cubic-diamond-cage structures, have unique properties with potential value for nanotechnology. The availability and ability to selectively functionalize this special class of nanodiamond materials opens new possibilities for surface-modification, for high-efficiency field emitters in molecular electronics, as seed crystals for diamond growth, or as robust mechanical coatings. The properties of self-assembled monolayers (SAMs) of diamondoids are thus of fundamental interest for a variety of emerging applications. This paper presents the effects of thiol substitution position and polymantane order on diamondoid SAMs on gold using nearedge X-ray absorption fine structure spectroscopy (NEXAFS) and X-ray photoelectron spectroscopy (XPS). A framework to determine both molecular tilt and twist through NEXAFS is presented and reveals highly ordered diamondoid SAMs, with the molecular orientation controlled by the thiol location. $\mathrm{C} 1 \mathrm{~s}$ and S $2 \mathrm{p}$ binding energies are lower in adamantane thiol than alkane thiols on gold by $0.67 \pm 0.05 \mathrm{eV}$ and $0.16 \pm 0.04 \mathrm{eV}$ respectively. These binding energies vary with diamondoid monolayer structure and thiol substitution position, consistent with different amounts of steric strain and electronic interaction with the substrate. This work demonstrates control over the assembly, in particular the orientational and electronic structure, providing a flexible design of surface properties with this exciting new class of diamond clusters.
\end{abstract}




\section{Introduction}

Diamondoids are hydrocarbon molecules with cubic-diamond-cage structures that have unique properties with potential value for nanotechnology. The lower diamondoids, with three or fewer diamond cages, are adamantane, diamantane, and triamantane. Higher diamondoids, nanometer-sized diamond molecules with greater than three diamond cages, have largely evaded laboratory synthesis ${ }^{1}$ and have only recently been purified from petroleum sources ${ }^{2}$. These diamondoids exhibit a variety of isomeric structures. For example, the four tetramantane isomers resemble the structures of a rod, left- and right- handed helices, and a trigonal platform. As the number of diamond cages increases, multiple molecular weights become possible within the same family and the numbers of structural isomers greatly expands. In contrast to larger diamond nanoparticles $(\sim 2.5 \mathrm{~nm})$, monodispersed diamondoids can be isolated in high purity and with isomeric selectivity ${ }^{2}$. The combination of a wide range of structures, high purity, and selective functionalization makes diamondoids an ideal platform for studies of diamond nanocluster surfaces, nano-mechanical properties, and quantum confinement.

Diamondoid electronic properties are an interesting blend between macroscopic diamond and small $\mathrm{sp}^{3}$-bonded hydrocarbon molecules ${ }^{3-5}$. The lowest unoccupied electronic states stem from the hydrogen surface termination and do not shift in energy as a function of $\operatorname{size}^{3}$, in contrast to $\mathrm{Si}$ and $\mathrm{Ge}^{6,7}$. The highest occupied states, however, exhibit clear size-dependent shifts ${ }^{5,8}$, similar to other group IV nanoparticles ${ }^{6,9}$. This demonstrates the ability to tune HOMO-LUMO gaps and other electronic properties based on diamondoid size ${ }^{10}$. Computations also support the observed quantum confinement effects $^{11-13}$ and have predicted negative electron affinities (NEAs) in some of the higher diamondoids ${ }^{12}$. Negative electron affinity and monochromatic electron emission have recently been demonstrated for [121] tetramantane-6-thiol (8, Figure 1) on $\mathrm{Au}$ and $\mathrm{Ag}$ surfaces ${ }^{14}$. In this case, the intimate connection between the metal and the diamondoid appears to play a key role in the emission process.

In order to take advantage of the unique properties that diamondoids offer, robust processing and handling techniques must be developed. Over the past two decades, SAMs of thiol-functionalized molecules on Au have emerged as one of the most convenient and widely used means for forming well-ordered films of small molecules ${ }^{15}$. With the recent success of site-specific functionalization of diamondoids ${ }^{16-20}$, it is now possible to use higher-diamondoids as molecular building blocks to yield materials with well-defined structures, including SAMs of higher-diamondoids. Self-assembled monolayers of adamantane-1-thiol, the smallest, most abundant, and most readily synthesized diamondoid, forms highly packed hexagonal layers on $\mathrm{Au}(111)$ reportedly with lower defect densities than alkane thiols, with a 7x7 reconstruction and $6.9 \AA$ nearest neighbor distance ${ }^{21-23}$. These adamantanethiol monolayers are also readily displaced by alkane thiols ${ }^{22,23}$, a useful property for nanolithography ${ }^{22-25}$. This labile behavior is attributed to the bulky nature of the adamantane which may impose steric restrictions that induce non-optimal $\mathrm{Au}-\mathrm{S}$ bonding ${ }^{23}$. Au-S bonding, and in particular, nature of $\mathrm{S}$ adsorption site is generating significant interest ${ }^{26-29}$, and with various experimental and theoretical results, "a single well-defined binding geometry may not necessarily be an appropriate description." 30 Based on geometric arguments, Au-S-C bond angles of the 
higher diamondoids are expected to vary depending upon the diamondoid order, functionalization site, and isomer geometry.

Near-edge X-ray Absorption Fine Structure (NEXAFS) powerfully determines molecular orientation on surfaces, but diamondoids present challenges to standard analysis techniques. Typically, molecular orientation is derived using a set of resonances in a plane or along a specific vector direction to determine a single parameter, usually the polar angle of the molecules ${ }^{31}$. Diamondoids, however, possess angular-dependent resonance intensities dependent upon both polar (tilt) and dihedral (twist) angle. Recently, additional functional groups attached to the end of aromatic SAMs were used to elegantly simultaneously determine both tilt and twist $^{32}$. Here, a more generalized framework for the "building block" scheme is presented, allowing for arbitrary choice of molecular axis and arbitrary molecule structure. In this framework, all possible tilts and twists consistent with the angular-dependent NEXAFS are compared with all sterically possible orientations ${ }^{33}$. The overlap between these two give all possible orientations within the accuracy and limitations of the building-block model ${ }^{31,34-36}$.

In this paper, SAMs formed from eight different diamondoid thiols on gold are investigated. Near-edge X-ray absorption fine structure spectroscopy (NEXAFS) determines the molecular orientation of the diamondoids on the surface, while X-ray photoelectron spectroscopy is used to investigate surface composition and the nature of the gold-sulfur bond. The NEXAFS results combined with sterically allowable tilt and twist angles provide a detailed characterization of the monolayers, and demonstrate control over diamondoid orientation through the position of the thiol. These results, combined with forthcoming complimentary measurements (e.g., STM) will provide a comprehensive characterization of diamondoid monolayers formed through self-assembly on gold. Variations in the $\mathrm{C} 1 \mathrm{~s}$ and $\mathrm{S} 2 \mathrm{p}$ binding energies are observed and depend on the thiolate environment and proximity to the gold substrate observed with NEXAFS. In particular, the $\mathrm{S} 2 \mathrm{p}$ variations indicate different sulfur configurations and potentially weaker Au-S bonds in diamondoid thiolates, in agreement with the previously reported higher lability of adamantane thiol ${ }^{22}$. The $S 2 p$ changes are also largest in prostrate monolayers, where NEXAFS in combination with steric considerations shows the largest deviation from the optimal gold-sulfur bonding.

\section{Experimental}

Diamondoids were extracted and purified from petroleum sources ${ }^{2}$, and the respective thiols 1-8 (Fig. 1) were prepared as described previously ${ }^{1,16-19}$.

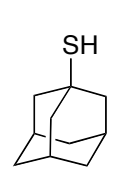

1

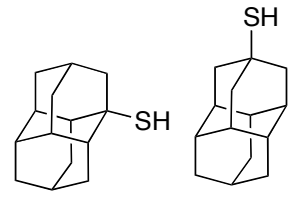

2

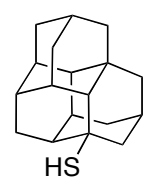

4

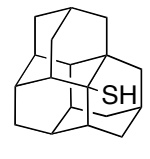

5

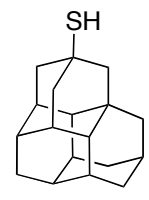

6

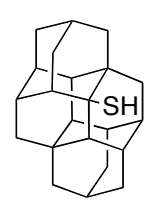

7

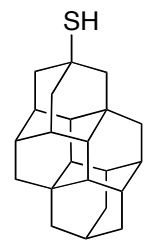

8

Figure 1. Diamondoid thiols prepared and used for the present study are 1: adamantanethiol, 2:diamantane-1-thiol, 3: diamantane-4-thiol, 4: triamantane-3-thiol, 5: triamantane-2-thiol, 6: triamantane9-thiol, 7: [121] tetramantane-2-thiol, and 8: [121]tetramantane-6-thiol. 
The diamondoid thiolate monolayers were prepared on $\mathrm{Au}(111)$ substrates via immersion in $1 \mathrm{mM}$ ethanolic solutions for 1-2 days. For some thiols, particularly structure 5 in Fig. 1, the addition of $10 \mathrm{vol} \%$ of toluene was necessary to aid in dissolving the thiols prior to dilution in ethanol. After removal from the diamondoid thiol solutions, samples were rinsed with clean ethanol, carefully dried with nitrogen, and quickly loaded into the vacuum chamber and pumped to ultra-high vacuum (UHV) below $\sim 10^{-9}$ torr to minimize exposure to ambient laboratory conditions ${ }^{37}$.

X-ray absorption and X-ray photoemission spectra were recorded on beamlines (BL) 8.2 and 10.1 of the Stanford Synchrotron Radiation Laboratory (SSRL, SPEARIII) at the Stanford Linear Accelerator (SLAC) ${ }^{38,39}$. The cross-section of the focused beam was approximately $1 \mathrm{~mm}$ in diameter on both 8.2 and 10.1 at the sample surface. NEXAFS spectra were recorded simultaneously in both total electron yield (TEY) and Auger electron yield (AEY) modes ${ }^{40}$. All NEXAFS signals were normalized to the $I_{0}$ current, which was recorded for the incident X-ray beam via a Au grid located upstream of the experimental sample. To ensure minimal effect on the $I_{0}$ signal from predominantly organic contaminants absorbed on the surface of the grid, it was frequently coated with a fresh layer of evaporated $\mathrm{Au}$. The $\pi^{*}$ resonance intensity in NEXAFS from freshly-cleaved highly oriented pyrolytic graphite (HOPG) served both as an energy calibration standard and as a measure of the degree of linear polarization, $P$, of the incident beam. HOPG incident angles were carefully selected such that comparison of the $\mathrm{C} \mathrm{K}$-edge $\pi^{*}$ resonance intensity yielded the relative magnitudes of $E_{p}{ }^{2}$ and $E_{s}{ }^{2}$, where $E_{p}$ and $E_{s}$ represent the electric field in-plane and perpendicular to the plane of incidence respectively ${ }^{40}$. $P$ was then calculated according to: ${ }^{31,41}$

\section{Equation 1 \\ $P=\frac{E_{p}{ }^{2}}{E_{p}{ }^{2}+E_{s}^{2}}$}

The calculated polarization was $99 \%$ in the plane of the storage ring for BL8.2, while the wiggler radiation from BL10.1 showed about $76 \%$ polarization during the course of the experiments presented in this paper. Care was taken to ensure that the effects of beam damage on the samples were minimized when conducting NEXAFS and PES measurements. Each spectrum was recorded from a fresh region of the sample surface and beam exposure during data collection was limited to the timeframe required for good signal to noise statistics.

All XPS data was recorded using the PHI15-255G CMA, which was operated at a pass energy of $25 \mathrm{eV}$. C 1s spectra were obtained at an incident photon energy of 400 $\mathrm{eV} ; \mathrm{S}(2 \mathrm{p})$ spectra were obtained at incident photon energies of 280 and $400 \mathrm{eV}$. For the purposes of energy calibration, a PES spectrum of the $\mathrm{Au} 4 \mathrm{f}$ electrons was recorded immediately after each $\mathrm{C} 1 \mathrm{~s}$ and $\mathrm{S} 2 \mathrm{p}$ measurement on the same region of the sample surface. The $\mathrm{Au} 4 \mathrm{f}_{7 / 2}$ photoelectron at $84.0 \mathrm{eV}^{42,43}$ was then used to convert from kinetic energy to binding energy scales. Furthermore, the C 1s and S $2 p$ spectra from freshly prepared dodecane thiolate monolayers on $\mathrm{Au}$ were recorded periodically to serve as absolute calibration standards. 


\section{Analysis}

NEXAFS yields a quantitative assignment of bond orientation, and hence molecular orientation for rigid structures such as the diamondoids. The intensity of a NEXAFS resonance is proportional to the dot product of the electric field vector in the Xray beam and the transition dipole moment (TDM) for the unoccupied orbital. By rotating the sample, one can vary the electric field to be completely in the surface plane at normal beam incidence, and nearly normal to the surface at grazing incidence. Analysis of NEXAFS resonance intensity as a function of incidence angle leads to the determination of molecular orientation.

Diamondoids present challenges to using a standard NEXAFS analysis. The diamondoids have highly symmetrical structures, and therefore, one single resonance or one single set of resonances in a particular plane cannot be used to determine molecular orientation. As will be shown, the NEXAFS spectra exhibit small but reproducible angular dependence. Obtaining a quantitative assessment of diamondoid orientation necessitates developing a general framework incorporating the "building-block" method for transitions into $\mathrm{C}-\mathrm{H} \sigma^{*}$ and $\mathrm{C}-\mathrm{C} \sigma^{*}$ states. In such a model, electronic transitions are modeled as dipoles originating from the $\mathrm{C} 1 \mathrm{~s}$ orbitals located at each carbon atomic position, and these transition dipoles are coincident with the axes of the $\mathrm{C}-\mathrm{H}$ bonds and $\mathrm{C}-\mathrm{C}$ bonds. This assumption can fail for extended alkanes where the angular dependencies of the $\mathrm{C}-\mathrm{C} \sigma^{*}$ transition dipoles are more accurately described as directed along the backbone of the hydrocarbon ${ }^{34,35}$, but even such a molecular orbital approach can lack accuracy for this and other resonances ${ }^{36}$. Computed X-ray absorption, also employed in this work $^{40,44}$, is useful to understanding how these various complex phenomena affect angular dependent resonances in alkanes and other molecules, but is highly qualitative. Unlike extended alkanes, these diamondoids are compact, rigid structures where the "building block" scheme provides a means to obtain an approximate orientation of the diamondoids that is more quantitative than simple inspection of the NEXAFS. Further, the "building-block" approximation of $\mathrm{C}-\mathrm{H} \sigma * / \mathrm{R} *$ resonances has widely and consistently been used to estimate orientation of molecules on surfaces ${ }^{31}$.

A molecular tilt and a molecular twist angle describe the orientation of a surfaceattached diamondoid as presented in figure 2. These parameters are defined with respect to a conveniently defined molecular axis. The first parameter, the tilt (also known as polar or colatitudal) angle $\alpha$ of the diamondoid is measured with respect to the $\mathrm{Au}(111)$ surface normal. The second parameter, the twist (or dihedral) angle $\beta$ describes the degree of rotation about the molecular axis. One cannot independently resolve tilt and twist angles from the NEXAFS data as acquired here. It is possible, however, to derive a manifold of all combinations of tilt and twist that are consistent with the angular dependence of the NEXAFS and are also sterically viable. 

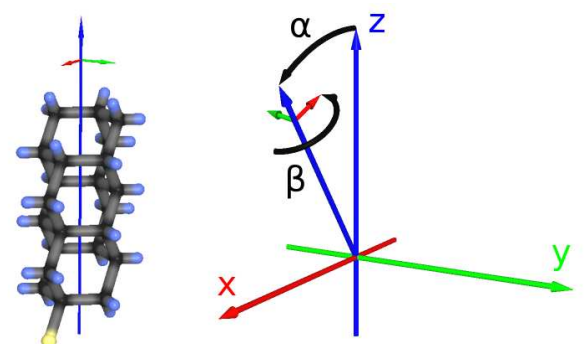

Figure 2: [left] One of the molecular axes chosen for 8, which lies along the [110] long-axis direction of the diamondoid. Transition dipole moments are defined with respect to this molecular axis; note the majority of $\mathrm{C}-\mathrm{H}$ bonds lie in planes orthogonal to this particular molecular z-axis. In contrast, most of the $\mathrm{C}-\mathrm{C}$ bonds lie generally along the axis. [right] The orientation of the molecule is defined by a tilt or polar angle $(\alpha)$ and a twist or dihedral angle $(\beta)$.

These parameters of tilt and twist are found from the angular dependence in the $\mathrm{X}$-ray absorption. The intensity of a resonance within a diamondoid can be written as the sum of the dot products squared of TDMs with the electric field of the incident radiation $^{31}$ :

\section{Equation 2}

$I=K \cdot \sum_{i}\left(\overrightarrow{\mathbf{E}} \cdot \overrightarrow{\mathbf{O}_{i}}\right)^{2}$

where $I$ is the intensity of the resonance, $\mathbf{E}$ is the electric field, and the $\mathbf{O}_{\mathbf{i}}$ are the transition dipole moments (TDMs). E can be represented in terms of the polarization $\mathrm{P}$, and the incident angle $\theta$ of the X-rays where $\theta=90^{\circ}$ is incident normal to the surface. A molecular axis is chosen, and the TDMs are then defined with respect to this molecular axis. These TDMs are denoted by M, with molecular orientation $\alpha, \beta$, and $\phi$ (the azimuthal orientation of the molecule with respect to the surface and $\mathrm{x}$-ray incidence plane $\left.^{31}\right)$. The intensity can then be written:

\section{Equation 3}

$$
\begin{aligned}
& I(\theta, \alpha, \beta, \phi)= \\
& K \cdot\left[\sum_{i}\left(\left[\begin{array}{c}
\sqrt{P} \cdot \sin (\theta) \\
\sqrt{1-P} \\
\sqrt{P} \cdot \cos (\theta)
\end{array}\right] \cdot\left[\begin{array}{ccc}
\cos (\phi) & -\sin (\phi) & 0 \\
\sin (\phi) & \cos (\phi) & 0 \\
0 & 0 & 1
\end{array}\right] \cdot\left[\begin{array}{ccc}
\cos (\alpha) & 0 & \sin (\alpha) \\
0 & 1 & 0 \\
-\sin (\alpha) & 0 & \cos (\alpha)
\end{array}\right] \cdot\left[\begin{array}{ccc}
\cos (\beta) & -\sin (\beta) & 0 \\
\sin (\beta) & \cos (\beta) & 0 \\
0 & 0 & 1
\end{array}\right] \cdot\left[\begin{array}{c}
M x_{i} \\
M y_{i} \\
M z_{i}
\end{array}\right]\right)\right]
\end{aligned}
$$


where the respective rotation matrices are used on the dipole moments with respect to the molecular axis. $\mathrm{K}$ is a proportionality constant that incorporates several scaling factors including the transition cross-section and the detection efficiency.

Several assumptions are incorporated into the analysis. First, the intensity variation as a function of incident angle arises from a large ensemble of molecules; a majority of these are assumed to have similar configuration on the surface. Structural inhomogeneity can bias the NEXAFS-derived result towards a magic angle ${ }^{31}$, or in this framework, a manifold of $\alpha$ and $\beta$ where no polarization dependence would be observed. For diamondoids, with multi-directional TDM's and at the observed monolayer purities, this effect will be minimal. Second, the azimuthal dependence can be averaged, since the domains are much smaller than the X-ray beam spot on the surface, and $\mathrm{Au}(111)$ nominally has three-fold symmetry, reducing equation 3 to:

\section{Equation 4}

$$
I(\Theta, \alpha, \beta)=K \cdot\left[\sum_{i}\left[(1-P \cdot \Theta) \cdot\left(G x_{i}^{2}+G y_{i}^{2}\right)+2 \cdot P \cdot \Theta \cdot G z_{i}^{2}\right]\right]
$$

where $\Theta=\cos ^{2}(\theta)$ and

\section{Equation 5}

$$
\left[\begin{array}{l}
G x_{i} \\
G y_{i} \\
G z_{i}
\end{array}\right]=\left[\begin{array}{ccc}
\cos (\alpha) & 0 & \sin (\alpha) \\
0 & 1 & 0 \\
-\sin (\alpha) & 0 & \cos (\alpha)
\end{array}\right] \cdot\left[\begin{array}{ccc}
\cos (\beta) & -\sin (\beta) & 0 \\
\sin (\beta) & \cos (\beta) & 0 \\
0 & 0 & 1
\end{array}\right] \cdot\left[\begin{array}{c}
M x_{i} \\
M y_{i} \\
M z_{i}
\end{array}\right]
$$

Note that equation 4 deconvolutes the resonances into an in-surface-plane component, and a surface-normal component, and each term in the sum reduces to the "vector case" presented as equation 9.16 in ref. ${ }^{31}$.

The experimental NEXAFS compared to intensities calculated using equations 4 and 5 determines plausible tilts and twists of the molecules on the surface. Polarizationdependent resonances of the experimental data are deconvoluted from the spectra using peakfitting. Ratios of various intensities to a particular incidence angle $\Theta_{\mathrm{r}}$, i.e.

\section{Equation 6}

$F(\Theta)=\frac{I(\Theta)}{I\left(\Theta_{r}\right)}$ 
normalize transition and detection cross-sections (factor $\mathrm{K}$ in equations 3 and 4), eliminating the need to explicitly determine these values ${ }^{31}$. The function $F(\Theta)$, using a fixed $\Theta_{\mathrm{r}}$, is a linear function of $\Theta$ (see equation 4). Linear regressions of the experimental data reduced to the form of equation 6 yield the slope of this line, along with $95 \%$ high and low confidence limits for this value ${ }^{45}$. The slope of $F(\Theta)$ can also be calculated for a given tilt and twist combination using equations 4 and 5 . All calculated slopes for various tilts and twists that lie within the $95 \%$ confidence limits represent possible diamondoid orientations.

A consideration of steric constraints determines the physically viable subset of the orientations obtained from linear regression analysis of the experimental NEXAFS. The acquired S $2 p$ core-level photoemission indicates that the diamondoidthiol is chemisorbed to the surface. With the sulfur fixed at the surface, feasible molecular tilt/twist combinations are those that have all atomic positions located above the surface plane. In contrast, any calculated diamondoid atomic position lying below the surface plane for a particular molecular tilt/twist constitutes a sterically impossible orientation. The intersection of sterically feasible and NEXAFS-derived orientations gives all possible combinations of tilt and twist for the surface-attached molecules.

\section{Results}

Figure 3 presents the NEXAFS data for the series of thiolated diamondoids on gold surfaces. NEXAFS spectra were acquired at $20^{\circ}$ (solid, red) $30^{\circ}$ (dashed, orange) $40^{\circ}$ (dashed, green) $55^{\circ}$ (dashed, cyan) $70^{\circ}$ (dashed, blue) and $90^{\circ}$ (solid, purple). The difference between the acquired spectra and the spectrum at $20^{\circ}$ is plotted just below the acquired spectra in order to accentuate the angular-dependent resonances. The small observed angular-dependencies are highly reproducible ${ }^{40}$. In all diamondoids, the $\mathrm{C}-\mathrm{H}$ $\sigma^{*} / \mathrm{R}^{*}$ resonances are present at about $287-289 \mathrm{eV}$ while the broad C-C $\sigma^{*}$ resonance is centered about $297 \mathrm{eV}^{31}$. These two manifolds of resonances exhibit angular dependence and are used to determine the molecular orientation. Although in some of the diamondoids, at least two $\mathrm{C}-\mathrm{H} \sigma^{*}$ resonances $^{3}$ are clearly resolvable (e.g. 6), the manifolds of these two surface terminations overlap and are thus considered together for all diamondoid thiols studied here. The series of diamondoids also exhibits the emergence of the bulk-diamond second gap band structure, characteristic of the diamondoids, at about $303 \mathrm{eV}^{3}$.

Analysis of the two longest diamondoids in this study, [121]tetramantane thiols 7 and $\mathbf{8}$, are presented as examples because they exhibit the strongest angular dependence. Tetramantanes have stronger intensity variation due to a prevalence of C-C bonds generally aligned with the long axis and a prevalence of bonds in a plane orthogonal to the long axis ( $\mathrm{C}-\mathrm{H}$ bonds) compared to smaller diamondoid thiols. Upon inspection, 8 has a $\mathrm{C}-\mathrm{H} \sigma^{*}$ resonance that is most intense at normal incidence and least intense at grazing incidence. Conversely, the $\mathrm{C}-\mathrm{C} \sigma^{*}$ resonance is most intense at grazing incidence and least intense at normal incidence. This is expected for an "upright" molecule (meaning the TDMs on average have a smaller polar angle than the magic angle ${ }^{31}$ ) on the surface. Comparable angular-dependent behavior is observed for alkane 
thiol SAMs on gold. In the case of 7, which has the thiol group attached at the [2]position on the side of the molecule, the opposite angular dependence is observed, with $\mathrm{C}-\mathrm{H} \sigma^{*}$ most intense at grazing incidence and the $\mathrm{C}-\mathrm{C} \sigma^{*}$ most intense at normal incidence. This indicates a prostrate orientation (meaning the TDMs on average have a larger polar angle than the magic angle) of this molecule on the surface. These qualitative assignments of orientation are consistent with angular dependence resonances of [121] tetramantane calculated with the StoBe DFT code. ${ }^{40,44}$ Qualitatively, the same effects are observed with the $\mathbf{3}$ and $\mathbf{6}$ (upright orientation) as well as $\mathbf{2}$ and $\mathbf{5}$ (prostrate orientation).

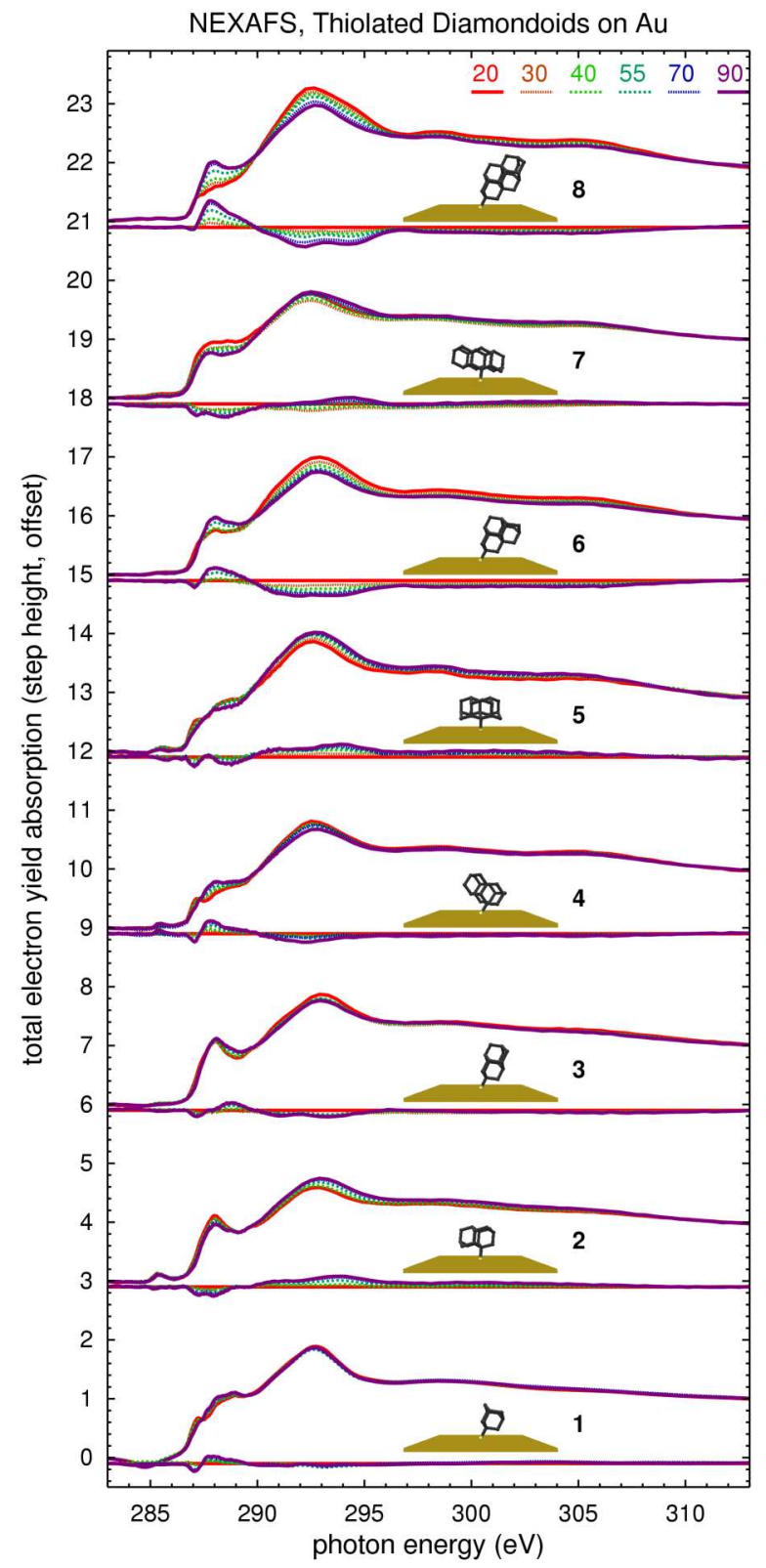

Figure 3: Near-edge X-ray absorption fine structure spectra for the series of diamondoid thiols 1-8. For each molecule, the NEXAFS traces at 20,30,40 55, 70 and $90^{\circ}$ incidence angle are shown. The lower traces have the $20^{\circ}$ trace subtracted to emphasize the angular dependence. 
A more quantitative estimate of orientation on the surface can be derived for 7 (tetramantane-2-thiol) and 8 (tetramantane-6-thiol). Figure 4 presents the normalized intensity of the $\mathrm{C}-\mathrm{H} \sigma^{*}$ resonance as a function of the cosine squared of the incidence angle for 8 (left pane) and 7 (right pane). The linear regression is presented for both cases, along with lines representing the 95\% confidence limits for the slope of the regression. This experimental parameter, the slope of normalized intensity vs. $\cos ^{2}(\theta)$, can also be simulated for a diamondoid of a given tilt and twist using the "buildingblock" approximation described above using equations 3 and 4 . The tilt/twist combinations yielding a simulated slope that lies within the $95 \%$ confidence limits of the experimental data are considered a viable orientation according to the NEXAFS. By combining these results with a geometric model of the sterically possible orientations, the actual orientations of the diamondoids can be estimated (Figures 5 and 6).
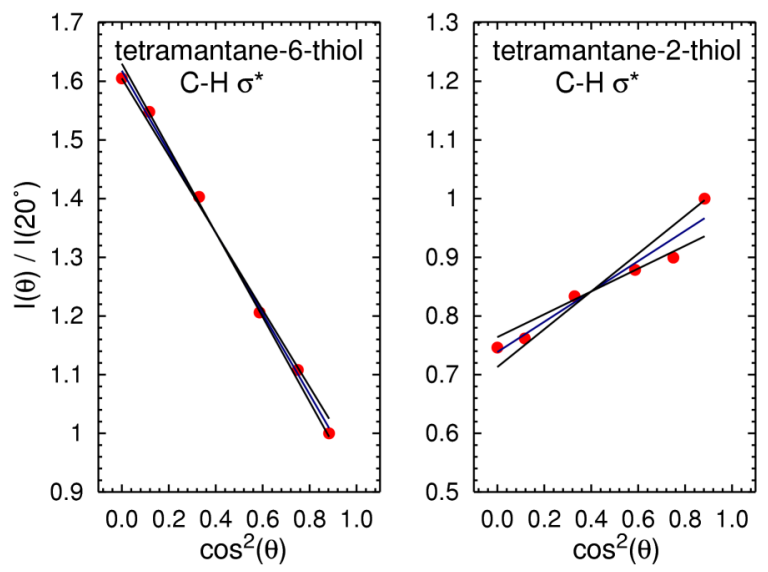

Figure 4: The ratios of normalized intensities of the resonances due to carbon-hydrogen bonds on gold plotted vs. the square of the incidence angle. The linear regression and 95\% confidence limits in the slopes are also plotted. Tetramantane-6-thiol (8) appears on the left, and tetramantane-2-thiol (7) is on the right.

Figure 5 graphically presents the possible orientations of $\mathbf{8}$ on gold. The top pane uses a molecular axis along the [110] long axis direction of these rod-like [121]tetramantane molecules. This allows for easy comparison with 7. The lower pane uses the sulfur-carbon bond as the molecular axis. Red areas show possible orientations from NEXAFS using the $\mathrm{C}-\mathrm{H} \sigma^{*}$ resonance; blue areas are derived from the $\mathrm{C}-\mathrm{C} \sigma^{*}$ resonance. In the cases presented here, the orientations derived from the $\mathrm{C}-\mathrm{H} \sigma^{*}$ overlap with those derived from the $\mathrm{C}-\mathrm{C} \sigma^{*}$. The gray regions of the plots represent sterically possible configurations of the molecule. Combinations of tilt and twist that yield slopes within the confidence limits of the NEXAFS but are sterically impossible are shown as faded red and blue. In both the upper and lower panes, pictoral representations of diamondoids in various possible orientations are presented, along with the corresponding molecular axes. Note that the figures in the upper and lower panes are identical with the exception of the molecular axes chosen to determine the configuration of the diamondoid on the surface.

Various tilts and twists are possible with respect to the long axis of $\mathbf{8}$ (Fig. 5, top pane); the tilt can vary from about $30^{\circ}$ to $60^{\circ}$ with a twist from about $-90^{\circ}$ to $+90^{\circ}$. In 
contrast, the plot in the lower pane demonstrates that the $\mathrm{S}-\mathrm{C}$ polar angle with respect to the surface is nearly constant at about $30 \pm 10^{\circ 14}$. Other diamondoids, where the sulfur is attached in a similar environment to adamantane thiol (3 and 6) also exhibit similar orientation, with larger uncertainty as these molecules exhibit relatively fewer $\mathrm{C}-\mathrm{C}$ bonds parallel to, and $\mathrm{C}-\mathrm{H}$ bonds perpendicular to the long molecular axis ${ }^{40}$. Further, qualitative comparison of NEXAFS compared to StoBe calculations performed on adamantanethiol (structure 1) are consistent with adamantanethiolate having this canted S-C bond on the surface. ${ }^{40,44}$
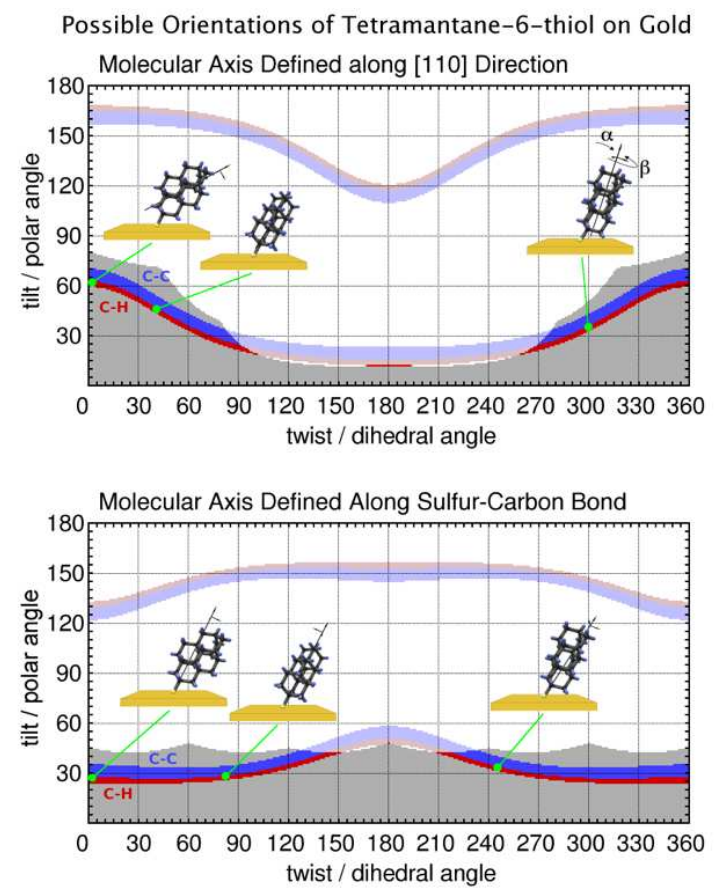

Figure 5: Possible tilts $(\alpha)$ and twists $(\beta)$ of [121]tetramantane-6-thiol (8) on $\mathrm{Au}(111)$. Top pane: molecular axis is defined along the [110] direction. Bottom pane: The molecular axis is defined along the sulfur-carbon bond. Sterically possible orientations are gray, while calculated NEXAFS within the 95\% confidence limits (cf. Figure 4) are shown in red and blue for the two resonances of interest. Pictorial representations of some of the orientations at different possible tilts and twists are also indicated.

Figure 6 presents the NEXAFS-derived possible orientations for 7 on gold. For simplicity, only one of four symmetrically equivalent thiol positions in this molecule is presented. In this case, the long-axis of the molecule is prostrate to the surface (tilt angle of $95 \pm 10^{\circ}$, upper pane) while the $\mathrm{S}-\mathrm{C}$ bond appears to be nearly normal to the surface $\left(10 \pm 10^{\circ}\right.$, lower pane). Structures 5 and $\mathbf{2}$ also exhibit similar prostrate configurations ${ }^{40}$.

In general, this set of diamondoid thiols generate monolayers on Au in which the monomers adopt upright configurations when the sulfur substitution position is at the tip of the molecule, with an canted $\mathrm{S}-\mathrm{C}$ polar angle $\left(30^{\circ} \pm 10^{\circ}\right.$ for 8$)$, and more prostrate configurations when the sulfur position is on the side of the molecule with an $\mathrm{S}-\mathrm{C}$ bond that is more normal to the surface. The exception is $\mathbf{4}$, which appears to have a more tilted $\mathrm{S}-\mathrm{C}$ bond angle and canted diamondoid orientation ${ }^{40}$. 

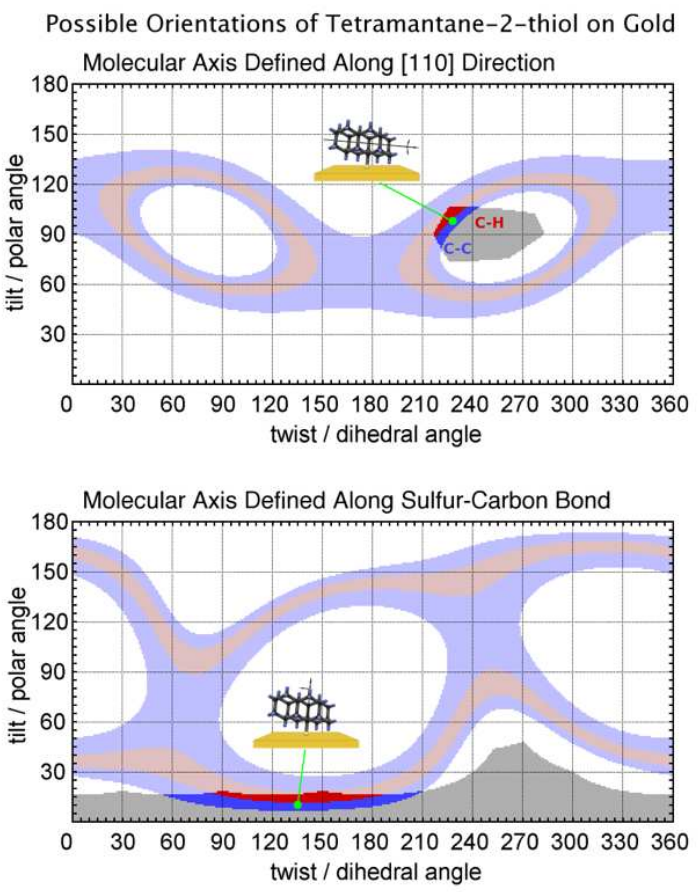

Figure 6: Possible tilts and twists of [121]tetramantane-2-thiol (7) on gold. Top pane: molecular axis is defined along the (110) direction. Bottom pane: molecular axis is defined along the sulfur-carbon bond. Sterically possible orientations are depicted with gray. Calculated NEXAFS resonances lying within the $95 \%$ confidence limits of the data (cf. Fig. 5) are presented in red and blue for the two resonances of interest.

Further information can be gained about the surface-attachment and structure of the monolayers through $\mathrm{C} 1 \mathrm{~s}$ and S $2 \mathrm{p}$ core-level XPS. Normalized Carbon $1 \mathrm{~s}$ and $\mathrm{Au} 4 \mathrm{f}$ XPS spectra for the series of diamondoid thiolates on $\mathrm{Au}$ as well as a reference SAM (dodecane thiolate/Au) are presented in figure 7. The gold photoelectron peaks (right pane) do not exhibit appreciable changes in shape or full-width at half maximum. These electrons are thus inferred to represent the bulk-gold, where the Fermi-level is fixed with respect to the electron analyzer, and are used to calibrate the binding energy scale of the other photoelectron spectra. Adamantane thiolate (1) displays a $\mathrm{C} 1 \mathrm{~s}$ binding energy that is $0.67 \pm 0.05 \mathrm{eV}$ lower than dodecane thiolate, in agreement with previous results ${ }^{23}$. This is much larger than the $0.3 \mathrm{eV}$ difference seen between unfunctionalized adamantane and cyclohexane ${ }^{8}$, indicating more than chemical shift contributions from structure alone. The S $2 p$ binding energy of adamantane thiolate also is lower than dodecane thiolate by $0.16 \pm 0.04$, as seen in Figure 8 . This small binding energy difference, not observed previously ${ }^{23}$, is resolvable using high-brightness, $280 \mathrm{eV}$ photons. 


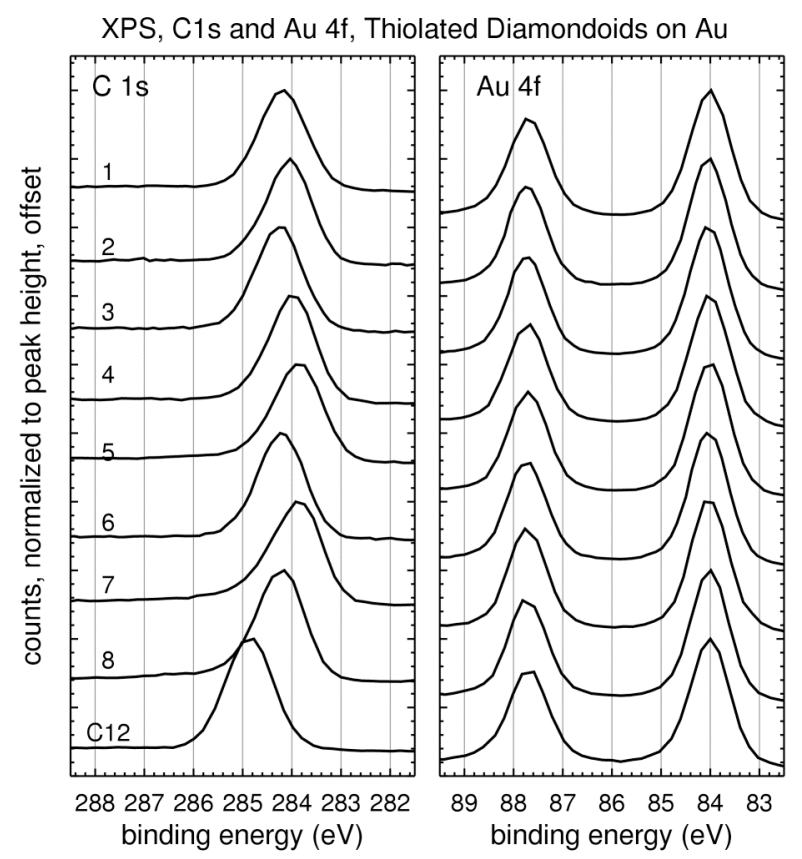

Figure 7: $\mathrm{C} 1 \mathrm{~s}$ and $\mathrm{Au} 4 \mathrm{f}$ XPS for the series of diamondoid thiolates 1-8 (see Fig. 1) and the corresponding photoelectron peaks acquired from dodecane thiolate/Au, labeled $\mathrm{C} 12$.

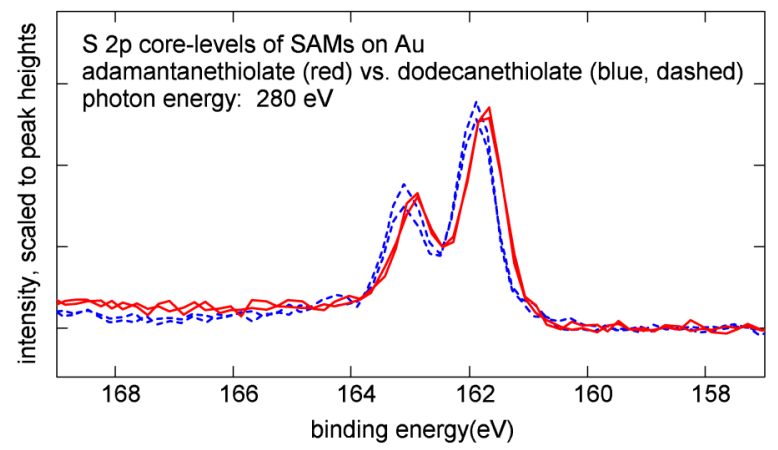

Figure 8: S 2p X-ray photoelectron spectra for adamantanethiolate (1) on gold (solid, red) and dodecane thiolate on gold (dashed, blue.) The S 2p binding energies for the diamondoid thiolate are about $0.16 \pm$ $0.04 \mathrm{eV}$ lower than the alkane thiolate on Au. A linear background has been subtracted, and peak heights are normalized to emphasize this small binding energy difference. Two acquisitions on each sample are presented.

Both carbon and sulfur photoelectrons exhibit binding energies that depend upon diamondoid and thiol attachment position. The $\mathrm{C} 1 \mathrm{~s}$ and $\mathrm{S} 2 \mathrm{p}$ binding energies referenced to a dodecane thiolate monolayer on gold, are summarized in Figure 9. Filled circles and error bars depict the $\mathrm{S} 2 \mathrm{p}_{3 / 2}$ binding energies, using the left axis to compare to dodecane thiolate on $\mathrm{Au}$, while fits to the $\mathrm{C} 1 \mathrm{~s}$ peak are also presented on this figure using the right axis. The data presented in this manner illustrates that the $\mathrm{C} 1 \mathrm{~s}$ and $\mathrm{S} 2 \mathrm{p}$ relative shifting in this series of diamondoids is in tandem. The $\mathrm{C} 1 \mathrm{~s}$ positions lie within the error of the $S 2 p$, perhaps with the exception of 4 . Structures $\mathbf{1}, \mathbf{3}, \mathbf{6}$, and $\mathbf{8}$ have very similar $\mathrm{C} 1 \mathrm{~s}$ binding energies and also $\mathrm{S} 2 \mathrm{p}$ binding energies. In these diamondoid thiols, the local environment around the SH groups are comparable. The remaining 
diamondoids, which all bear the thiol substituent at the side of the molecule, have lower binding energies. For sulfur, the larger error bars for $\mathbf{5}$ and $\mathbf{7}$ are due to difficulty in deconvoluting the prominent gold-thiolate component from various residual sulfur species also present in the monolayer. In particular, $\mathbf{5}$ is difficult to dissolve in solution, resulting in layers with an unbound thiol/disulfide component of varying intensity. Occasionally, this and some of the other diamondoid monolayers also exhibit a small doublet having S 2 $\mathrm{p}_{3 / 2}$ binding energy of about $161 \mathrm{eV}$, presumably (but possibly not uniquely $^{46}$ ) due to trace elemental sulfur contaminants on the Au surface ${ }^{47-49}$.

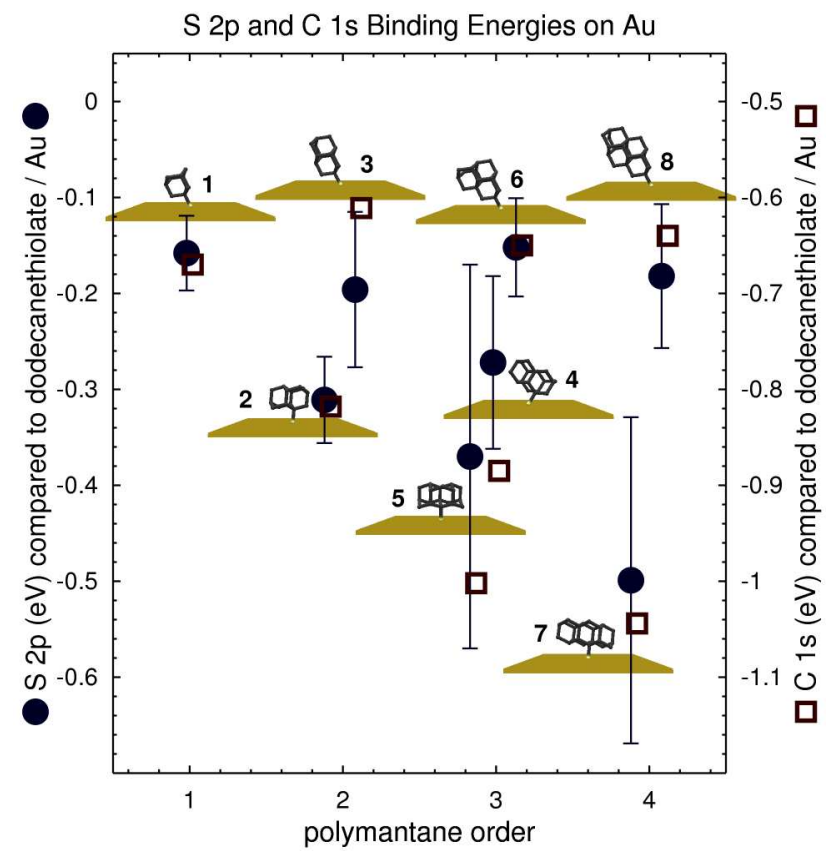

Figure 9: The core-level binding energies of the $S 2 p$ and $C 1 s$ photoelectrons relative to dodecane thiol on $\mathrm{Au}$. S 2p binding energies are depicted filled circles, using the left axis, while $\mathrm{C}$ 1s binding energies are depicted with open boxes and are plotted using the right axis. Multiple sulfur sites for a given diamondoid are offset for clarity. Figures depict orientations derived with NEXAFS for each diamondoid thiolate monolayer.

\section{Discussion}

The NEXAFS data shows diamondoid thiolates on $\mathrm{Au}(111)$ exhibit upright or prostrate orientation that is dependent upon the thiol substitution position. Using a quantitative model, under the assumption that most molecules in a particular monolayer assume similar configurations on the surface, diamondoids with a thiol moiety at the end or apical position $(\mathbf{1}, \mathbf{3}, \mathbf{6}$, and 8) assume an upright orientation with the sulfur-carbon bond tilted with a polar angle of about $30^{\circ}$. These four consistently form the most chemically homogeneous (i.e. one S2p doublet) monolayers. Alternatively, the diamondoid thiols with thiol substitution at positions other than the very end of the molecule, 2, 5, and 7, adopt a prostrate orientation, with the sulfur-carbon bond nearly normal to the $\mathrm{Au}$ surface. The exception is 4 , which assumes a more tilted $\mathrm{S}-\mathrm{C}$ bond angle than 2, 5, or 7. Pictorial representations of diamondoids in Figures 3 and 9 represent these derived orientations; note that diamondoids attached at apical positions could also be rotated around the S-C bond, as depicted for 8 in Figure 5. 
None of these diamondoid monolayers allow sulfur to be in the nominal configuration seen in alkane or other thiol-based SAMs on gold. Noting results of goldsulfur bonding mechanisms vary greatly, ${ }^{26-30}$, experimental studies of thiolate SAMs on gold illustrate the $\mathrm{Au}-\mathrm{S}-\mathrm{C}$ bond angle is about $104-110^{\circ}$, corresponding to an $\mathrm{S}-\mathrm{C}$ polar angle of about $70-76^{\circ}$ with respect to the surface normal ${ }^{47,50-54}$ while STM displays methanethiolate adsorbing on gold with "strongly tilted absorption configuration"28. In contrast, these NEXAFS results show diamondoid thiolates with $\mathrm{S}-\mathrm{C}$ bonds ranging from near the surface normal to about $30^{\circ}$. The apical cases of $\mathbf{1 , 3}, \mathbf{6}$, and $\mathbf{8}$ are oriented near the steric limit, where diamondoid hydrogen atoms near the Au surface prevent the S-C bond from assuming a configuration similar to other thiols. The strain induced in the Au$\mathrm{S}$ bond in apical cases can lead to a weaker bond to the substrate, as seen in adamantane thiol $^{22}$. Even greater strain in diamondoids such as 2, 5, and 7 may affect the strength of the Au-S bond even further. Although 7 could assume a more canted S-C bond similar to apical cases (cf. Fig. 6, lower pane, twist angle of $\sim 270^{\circ}$ ) the NEXAFS does not overlap this sterically accessible region. In the twist angles where NEXAFS overlaps $\left(60-210^{\circ}\right)$ the diamondoid resides in a more sterically restricted environment, with maximum possible tilt of about $15^{\circ}$.

Carbon and sulfur photoemission peak energy differences are correlated to orientation derived through the NEXAFS. A number of initial- and final- state effects contribute to the $\sim 0.6 \mathrm{eV}$ constant offset in $\mathrm{C} 1 \mathrm{~s}$ binding energy between alkane and diamondoid $\mathrm{SAMs}^{8,23,55}$, but most interesting is the variation between diamondoid thiolates on gold. The apparent shift to lower binding energy in the $\mathrm{C} 1 \mathrm{~s}$ is highly correlated to how prostrate the molecule lies on the surface. An indicator of how prostrate the molecule lies is the number of hydrogen atoms in close proximity to the surface based on NEXAFS-derived orientations; i.e. about two hydrogens for $\mathbf{1}, \mathbf{3}, \mathbf{6}$, and 8 (0.61 to $0.65 \mathrm{eV}$ lower than dodecane thiol) three for $\mathbf{2}$ and 4, $(-0.82$ and $-0.89 \mathrm{eV})$ and four hydrogens for 5 and $7(-1.00$ and $-1.04 \mathrm{eV})$. Alkane thiols on gold are not Fermi level aligned, but rather have vacuum level alignment ${ }^{56}$ and similar behavior appears to affect the diamondoids. Thus the simplest hypothesis for the differing $\mathrm{C} 1 \mathrm{~s}$ is that the diamondoid lies at a slightly different potential than the gold substrate, with the misalignment of Fermi levels dependent upon the proximity of the diamondoid to the gold. The varying $\mathrm{C}$ 1s energy also indicates that the electronic interaction between the diamondoid and substrate can be tuned based on the location of the thiol position. Although the $\mathrm{C} 1 \mathrm{~s}$ and $\mathrm{S} 2 \mathrm{p}$ change in tandem, and this diamondoid data alone cannot prove otherwise, the precedent in alkane thiols show that $\mathrm{C} 1 \mathrm{~s}$ and $\mathrm{S} 2 \mathrm{p}$ shifting arise from two independent processes. Prostrate alkanethiols observed in well-known striped phases during early stages of SAM growth have C 1s and S $2 p$ binding energies that are both about $0.6 \mathrm{eV}$ lower than in well-packed, upright SAMs ${ }^{57,58}$; however, during SAM formation, the shifting towards higher binding energy occur earlier in the $\mathrm{S} 2 \mathrm{p}$ than the $\mathrm{C}$ $1 \mathrm{~s}$, implying different, independent mechanisms ${ }^{58}$.

The differences in binding energy of the sulfur $2 p$ among the diamondoid thiolates as well as the lower binding energy compared to dodecane thiolate on gold are consistent with the strained Au-S bonds observed with NEXAFS. A portion of the $0.16 \pm 0.04 \mathrm{eV}$ binding energy shift seen in adamantane thiolate (and similar higher diamondoid thiolates) relative to alkane thiolates is likely due to dissimilar sulfur nextnearest neighbor environments: in diamondoid thiols the sulfur is bound to a carbon that 
is bound to three other carbon atoms, while in the alkanethiols, the sulfur is bound to an alkyl unit (e.g. dodecane-1-thiol). In addition, strain in $\mathrm{Au}-\mathrm{S}-\mathrm{C}$ angle reduces the $\mathrm{S} 2 \mathrm{p}$ binding energy ${ }^{52-54}$. Structures $\mathbf{1}, \mathbf{3}, \mathbf{6}$, and $\mathbf{8}$, with similar local sulfur environments, cannot assume the nominal, highly canted S-C polar angle of 70-76 ${ }^{\circ 2}$ observed for the alkane thiols (e.g., the lower pane of Figure 5). The S-C bond is strained, and potentially elongated and weaker than a gold-alkanethiolate bond. Further, in molecules where NEXAFS indicates this bond is closer to the surface normal (especially in $\mathbf{2 , 5}$, and $\mathbf{7}$ ) the $\mathrm{S} 2 \mathrm{p}$ binding energy is even further reduced and supports even larger S-Au bond strain in these cases. Alkane thiols are reported to adsorb on silver with an S-C polar angle that is nearly normal to the surface ${ }^{51}$. Work has commenced to ascertain whether these binding energy shifts attributed to strain are eliminated when the diamondoid thiols are adsorbed on Ag where the nominal bonding configuration of the sulfur observed in alkanethiol SAMs can be attained with diamondoid thiols.

A number of other interesting issues are not resolved with NEXAFS and XPS alone. For example, structure 7 may have a complex or even incommensurate relationship to the underlying gold lattice. Once chemisorbed on the surface this molecule exhibits two enantiomers, which could presumably lead to a lack of order, i.e., an irregular surface-pattern. This does not eliminate the potential for orientational similarity. One of the major assumptions made in interpreting the NEXAFS is that each diamondoid in a monolayer adsorbs in similar orientational conformation, and this is not yet evident that this is the case. Even in the other diamondoid thiols, additional STM results will be valuable in confirming this assumption.

The NEXAFS results demonstrate tunability in which analogue of the crystallographic faces of bulk diamond is exposed at the surface of the monolayer. This could have impact in, for example, crystal growth or in building nanoscale assemblies using diamondoids as "molecular building blocks." Monolayers formed on gold with 2, $\mathbf{5}$, and 7, with the $\mathrm{S}-\mathrm{C}$ bond nearly normal to the surface, present close to a diamond(111)-like surface. The triamantane-3-thiolate, with a more canted Au-S-C angle, presents close to a (110) surface $^{40}$. For $\mathbf{1}, \mathbf{3}, \mathbf{6}$, and $\mathbf{8}$, the crystallographic plane at each monolayer surface cannot be uniquely determined at this time due to the range of possible dihedral angles the NEXAFS indicates for these surface-attached diamondoid thiol monomers.

The results presented herein indicate clean, orientationally ordered films obtained from a wide variety of diamondoid thiols. They also indicate that in diamondoid thiols, the anchoring bond to the Au surface may be in a different configuration compared to other types of thiol-based self-assembled monolayers on gold. This tunability of $\mathrm{Au}-\mathrm{S}$ bond configuration, however, may be useful in nanolithography or other applications where varying the strength of substrate-molecule bonding is desirable, for example in substitution reactions. Characterization of these phenomena in diamondoid thiols adsorbed on surfaces provides understanding that is of fundamental importance in the development and optimization of robust monolayers for technological applications.

\section{Conclusions}


Control over the orientation of diamondoid monolayers using selectively thiolated diamondoids adamantane, diamantane, triamantane, and [121]tetramantane on gold has been demonstrated. A general method, presented in this work, determines plausible tilts and twists for arbitrary molecules from NEXAFS data. This method reveals orientationally ordered diamondoid SAMs, with the molecular orientation controlled by the thiol attachment point. Through the analysis of NEXAFS spectra, the sulfur-carbon bond exhibits a polar angle of about $30^{\circ} \pm 10^{\circ}$ degrees in [121]tetramantane-6-thiol (structure 8). Adamantane, diamantane, triamantane thiolated at similar apical positions $(\mathbf{1}, \mathbf{3}$, and $\mathbf{6})$ assume comparable upright orientations with canted sulfur-carbon polar angles. In contrast, diamondoids with thiols positioned at the side in $\mathbf{2 , 5}$, and $\mathbf{7}$ lead to prostrate orientation, where the sulfur-carbon bond is more normal to the surface than in the apical thiol cases. These $\mathrm{Au}-\mathrm{S}-\mathrm{C}$ bond angles are not in the lowest energy configuration seen in alkanethiols (e.g., $104-110^{\circ}$, corresponding to an $\mathrm{S}-\mathrm{C}$ polar angle of $70-76^{\circ}$ ) and thus appear strained. $\mathrm{C} 1 \mathrm{~s}$ and $\mathrm{S} 2 \mathrm{p}$ binding energies are lower in adamantanethiol than alkanethiols on gold by $0.67 \pm 0.05 \mathrm{eV}$ and $0.16 \pm 0.04 \mathrm{eV}$ respectively. These binding energies vary among the diamondoid thiolates, an indication of variable electronic interaction with the Au substrate. The differences in binding energy are hypothesized to arise from the following mechanisms: First, the $\mathrm{C} 1 \mathrm{~s}$ shifts to lower binding energy are roughly proportional to the number of hydrogen atoms in close proximity to the gold, causing a misalignment of Fermi levels between the gold and carbon frameworks of diamondoids in the monolayer. Second, the S $2 p$ shifting is consistent with the strained nature of the gold-thiolate bond: Prostrate diamondoid thiols where NEXAFS shows the $\mathrm{S}-\mathrm{C}$ bonds to be nearly normal to the surface exhibit the largest shifts towards lower S $2 p$ binding energies. This work demonstrates control over the assembly, in particular the orientational and electronic structure, and therefore the surface properties of this exciting new class of diamond nanoclusters.

\section{Supporting Information Available}

Additional NEXAFS data, derived tilt/twist plots, XPS spectra and fits, molecular axis definitions for each molecule, supporting StoBe DFT calculations, and experimental details are available in the supporting information. This information is available free of charge via the Internet at http://pubs.acs.org/.

\section{Acknowledgements}

We acknowledge Steven Horn, LLNL \& The Univ. of Lethbridge, Canada. We thank the staff of SSRL especially Jan Lüning, Dan Brehmer, and Curtis Troxel. This work was funded by the Office of Basic Energy Sciences, Materials Sciences, U. S. Department of Energy. Research was performed at the Stanford Synchrotron Radiation Laboratory, a national user facility operated by Stanford University on behalf of the U.S. D.O.E., Office of Basic Energy Sciences. This work was partially performed under the auspices of the U.S. Department of Energy by Lawrence Livermore National Laboratory under Contracts W-7405-Eng-48 and DE-AC52-07NA27344. Work performed at the Justus-Liebig University was supported by the Fonds der Chemischen Industrie. 


\section{References}

(1) Schwertfeger, H.; Fokin, A. A.; Schreiner, P. R. Angew. Chem. Int. Ed. 2008, 47, 1022-1036.

(2) Dahl, J. E.; Liu, S. G.; Carlson, R. M. K. Science 2003, 299, 96-99.

(3) Willey, T. M.; Bostedt, C.; van Buuren, T.; Dahl, J. E.; Liu, S. G.; Carlson, R. M. K.; Terminello, L. J.; Moller, T. Phys. Rev. Lett. 2005, 95, 113401.

(4) Wang, Y.; Kioupakis, E.; Lu, X.; Wegner, D.; Yamachika, R.; Dahl, J. E.; Carlson, R. M. K.; Louie, S. G.; Crommie, M. F. Nat. Mater. 2008, 7, 38-42.

(5) Willey, T. M.; Bostedt, C.; van Buuren, T.; Dahl, J. E.; Liu, S. G.; Carlson, R. M. K.; Meulenberg, R. W.; Nelson, E. J.; Terminello, L. J. Phys. Rev. B 2006, $74,205432$.

(6) van Buuren, T.; Dinh, L. N.; Chase, L. L.; Siekhaus, W. J.; Terminello, L. J. Phys. Rev. Lett. 1998, 80, 3803-3806.

(7) Bostedt, C.; van Buuren, T.; Willey, T. M.; Franco, N.; Terminello, L. J.; Heske, C.; Moller, T. Appl. Phys. Lett. 2004, 84, 4056-4058.

(8) Klünder, K., Photoelectron Spectroscopy on Molecular Diamond; Diploma Thesis, Technical University of Berlin, 2007.

(9) Williamson, A. J.; Bostedt, C.; van Buuren, T.; Willey, T. M.; Terminello, L. J.; Galli, G. Nano Lett. 2004, 4, 1041-1045.

(10) Lenzke, K.; Landt, L.; Hoener, M.; Thomas, H.; Dahl, J. E.; Liu, S. G.; Carlson, R. M. K.; Moller, T.; Bostedt, C. J. Chem. Phys. 2007, 127, 084320.

(11) McIntosh, G. C.; Yoon, M.; Berber, S.; Tomanek, D. Phys. Rev. B 2004, $70,045401$.

(12) Drummond, N. D.; Williamson, A. J.; Needs, R. J.; Galli, G. Phys. Rev. Lett. 2005, 95, 096801.

(13) Lu, A. J.; Pan, B. C.; Han, J. G. Phys. Rev. B 2005, 72, 035447.

(14) Yang, W. L.; Fabbri, J. D.; Willey, T. M.; Lee, J. R. I.; Dahl, J. E.; Carlson, R. M. K.; Schreiner, P. R.; Fokin, A. A.; Tkachenko, B. A.; Fokina, N. A.; Meevasana, W.; Manella, N.; Tanaka, K.; Zhou, X. J.; van Buuren, T.; Kelly, M. A.; Hussain, Z.; Melosh, N. A.; Shen, Z. X. Science 2007, 316, 1460-1462.

(15) Love, J. C.; Estroff, L. A.; Kriebel, J. K.; Nuzzo, R. G.; Whitesides, G. M. Chem. Rev. 2005, 105, 1103-1169.

(16) Tkachenko, B. A.; Fokina, N. A.; Chernish, L. V.; Dahl, J. E. P.; Liu, S. G.; Carlson, R. M. K.; Fokin, A. A.; Schreiner, P. R. Organic Letters 2006, 8, 1767-1770.

(17) Schreiner, P. R.; Fokina, N. A.; Tkachenko, B. A.; Hausmann, H.; Serafin, M.; Dahl, J. E. P.; Liu, S. G.; Carlson, R. M. K.; Fokin, A. A. Journal of Organic Chemistry 2006, 71, 6709-6720.

(18) Fokin, A. A.; Schreiner, P. R.; Fokina, N. A.; Tkachenko, B. A.; Hausmann, H.; Serafin, M.; Dahl, J. E. P.; Liu, S. G.; Carlson, R. M. K. J. Org. Chem. 2006, 71, 8532-8540.

(19) Fokin, A. A.; Tkachenko, B. A.; Gunchenko, P. A.; Gusev, D. V.; Schreiner, P. R. Chem. Eur. J. 2005, 11, 7091-7101.

(20) Kozhushkov, S. I.; Yufit, D. S.; Boese, R.; Blaser, D.; Schreiner, P. R.; de Meijere, A. Eur. J. Org. Chem. 2005, 1409-1415.

(21) Fujii, S.; Akiba, U.; Fujihira, M. JACS 2002, 124, 13629-13635. 
(22) Dameron, A. A.; Charles, L. F.; Weiss, P. S. JACS 2005, 127, 8697-8704.

(23) Dameron, A. A.; Mullen, T. J.; Hengstebeck, R. W.; Saavedra, H. M.; Weiss, P. S. J. Phys. Chem. C 2007, 111, 6747-6752.

(24) Dameron, A. A.; Hampton, J. R.; Gillmor, S. D.; Hohman, J. N.; Weiss, P. S. J. Vac. Sci. Technol., B 2005, 23, 2929-2932.

(25) Dameron, A. A.; Hampton, J. R.; Smith, R. K.; Mullen, T. J.; Gillmor, S. D.; Weiss, P. S. Nano Lett. 2005, 5, 1834-1837.

(26) Jadzinsky, P. D.; Calero, G.; Ackerson, C. J.; Bushnell, D. A.; Kornberg, R. D. Science 2007, 318, 430-433.

(27) Maksymovych, P.; Sorescu, D. C.; Yates, J. T. Phys. Rev. Lett. 2006, 97, -.

(28) Maksymovych, P.; Sorescu, D. C.; Yates, J. T. J. Phys. Chem. B 2006, $110,21161-21167$.

(29) Vericat, C.; Vela, M. E.; Benitez, G. A.; Gago, J. A. M.; Torrelles, X.; Salvarezza, R. C. J. Phys. Condens. Matter 2006, 18, R867-R900.

(30) Mazzarello, R.; Cossaro, A.; Verdini, A.; Rousseau, R.; Casalis, L.; Danisman, M. F.; Floreano, L.; Scandolo, S.; Morgante, A.; Scoles, G. Phys. Rev. Lett. 2007, 98, -.

(31) Stöhr, J. Nexafs Spectroscopy; Springer-Verlag: Berlin - Heidelberg - New York, 1992.

(32) Ballav, N.; Schüpbach, B.; Dethloff, O.; Feulner, P.; Terfort, A.; Zharnikov, M. JACS 2007, 129, 15416-15417.

(33) Lee, J. R. I.; Han, T. Y. J.; Willey, T. M.; Wang, D.; Meulenberg, R. W.; Nilsson, J.; Dove, P. M.; Terminello, L. J.; van Buuren, T.; De Yoreo, J. J. JACS 2007, 129, 10370-10381.

(34) Hähner, G.; Kinzler, M.; Wöll, C.; Grunze, M.; K. Scheller, M.; Cederbaum, L. S. Physical Review Letters 1991, 67, 851.

(35) Fu, J. X.; Urquhart, S. G. J. Phys. Chem. A 2005, 109, 11724-11732.

(36) Zou, Y.; Araki, T.; Appel, G.; Kilcoyne, A. L. D.; Ade, H. Chem. Phys. Lett. 2006, 430, 287-292.

(37) Willey, T. M.; Vance, A. L.; van Buuren, T.; Bostedt, C.; Terminello, L. J.; Fadley, C. S. Surf. Sci. 2005, 576, 188-196.

(38) Tirsell, K. G.; Karpenko, V. P. Nuclear Instruments \& Methods in Physics Research Section a-Accelerators Spectrometers Detectors and Associated Equipment 1990, 291, 511-517.

(39) Karpenko, V.; Kinney, J. H.; Kulkarni, S.; Neufeld, K.; Poppe, C.; Tirsell, K. G.; Wong, J.; Cerino, J.; Troxel, T.; Yang, J. Rev. Sci. Instrum. 1989, 60, 1451-1456.

(40) See Supporting Information, available at http://pubs.acs.org/

(41) Stohr, J.; Outka, D. A. Phys. Rev. B 1987, 36, 7891-7905.

(42) Seah, M. P.; Gilmore, L. S.; Beamson, G. Surface and Interface Analysis 1998, 26, 642-649.

(43) Seah, M. P.; Gilmore, I. S.; Spencer, S. J. Surface and Interface Analysis 1998, 26, 617-641.

(44) Hermann, K.; Pettersson, L. G. M.et al. StoBe-deMon version 3.0, 2007

(45) Taylor, J. R. An Introduction to Error Analysis: The Study of Uncertainties in Physical Measurements; University Science Books, 1982. 
(46) Shaporenko, A.; Terfort, A.; Grunze, M.; Zharnikov, M. J. Electron. Spectrosc. Relat. Phenom. 2006, 151, 45-51.

(47) Laibinis, P. E.; Whitesides, G. M.; Allara, D. L.; Tao, Y.-T.; Parikh, A. N.; Nuzzo, R. G. JACS 1991, 113, 7152-7162.

(48) Ishida, T.; Choi, N.; Mizutani, W.; Tokumoto, H.; Kojima, I.; Azehara, H.;

Hokari, H.; Akiba, U.; Fujihira, M. Langmuir 1999, 15, 6799-6806.

(49) Yang, Y. W.; Fan, L. J. Langmuir 2002, 18, 1157-1164.

(50) Nuzzo, R. G.; Dubois, L. H.; Allara, D. L. JACS 1990, 112, 558-569.

(51) Walczak, M. M.; Chung, C. K.; Stole, S. M.; Widrig, C. A.; Porter, M. D. JACS 1991, 113, 2370-2378.

(52) Heister, K.; Rong, H. T.; Buck, M.; Zharnikov, M.; Grunze, M.; Johansson, L. S. O. J. Phys. Chem. B 2001, 105, 6888-6894.

(53) Shaporenko, A.; Brunnbauer, M.; Terfort, A.; Grunze, M.; Zharnikov, M. J. Phys. Chem. B 2004, 108, 14462-14469.

(54) Shaporenko, A.; Brunnbauer, M.; Terfort, A.; Johansson, L. S. O.; Grunze, M.; Zharnikov, M. Langmuir 2005, 21, 4370-4375.

(55) Heister, K.; Johansson, L. S. O.; Grunze, M.; Zharnikov, M. Surf. Sci. 2003, 529, 36-46.

(56) Ahn, H.; Zharnikov, M.; Whitten, J. E. Chem. Phys. Lett. 2006, 428, 283287.

(57) Himmel, H. J.; Woll, C.; Gerlach, R.; Polanski, G.; Rubahn, H. G. Langmuir 1997, 13, 602-605.

(58) Himmelhaus, M.; Gauss, I.; Buck, M.; Eisert, F.; Woll, C.; Grunze, M. J. Electron. Spectrosc. Relat. Phenom. 1998, 92, 139-149. 
Table of contents graphic:

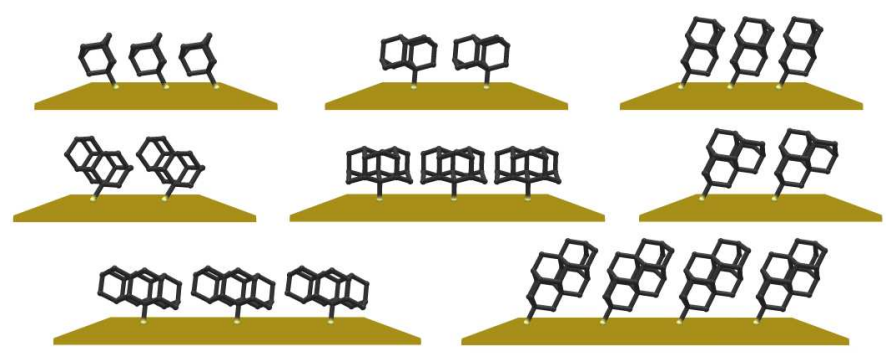




\section{Supporting Information}

\section{Near-Edge X-ray Absorption Fine Structure Spectroscopy of Diamondoid Thiol Monolayers on Gold}

Trevor M. Willey ${ }^{1 *}$, Jason D. Fabbri ${ }^{2}$, Jonathan R. I. Lee ${ }^{1}$, Peter R. Schreiner ${ }^{3}$, Andrey A. Fokin ${ }^{3}$, Boryslav A. Tkachenko ${ }^{3}$, Nataliya A. Fokina ${ }^{3}$, Jeremy E. P. Dahl ${ }^{4}$,

Robert M. K. Carlson ${ }^{4}$, Andrew L. Vance ${ }^{5}$, Wanli Yang ${ }^{6}$, Louis J. Terminello ${ }^{1}$, Tony van Buuren ${ }^{1}$, and Nicolas A. Melosh ${ }^{2}$

1) Materials Science and Technology Division, Lawrence Livermore National Laboratory, 7000 East Avenue, Livermore CA 94550

2) Materials Science and Engineering, Stanford University, 476 Lomita Mall, Stanford, CA 94305

3) Institute of Organic Chemistry, Justus-Liebig University Giessen, Heinrich-Buff-Ring 58, 35392 Giessen, Germany

4) MolecularDiamond Technologies, Chevron Technology Ventures, 100 Chevron Way, Richmond, CA 94802

5) Materials Chemistry Department, Sandia National Laboratories, 7011 East Avenue, Livermore, CA 94550

6) Advanced Light Source, Lawrence Berkeley National Laboratory, 1 Cyclotron Road, Berkeley, CA 94720 


\section{Additional NEXAFS Data}

The following pages present multiple NEXAFS acquisitions on thiolated derivatives of diamantane through tetramantane to show qualitative reproducibility. Each dataset is annotated with the approximate date and the beamline on which it was acquired at SSRL. Total electron yield is on the left, while Auger electron yield is on the right. The best two datasets of each sample were used to calculate the potential orientations. There are noticeable differences in data from the two beamlines: first, the polarization of 8.2 is about $99 \%$ in the plane of the storage ring, while at 10.1 , this value drops to about $76 \%$. Thus the angular dependence of resonance intensity is higher at 8.2 than 10.1. The grid was difficult to keep clean on 10.1, so some spurious carbon features can be observed, and beam damage also occurs much more readily on 10.1, a wiggler beamline. The same color scheme in the text is used: red is 20 degrees (grazing incidence) progressing through 30, 40, 55, 70 and 90 degrees in violet (normal incidence).
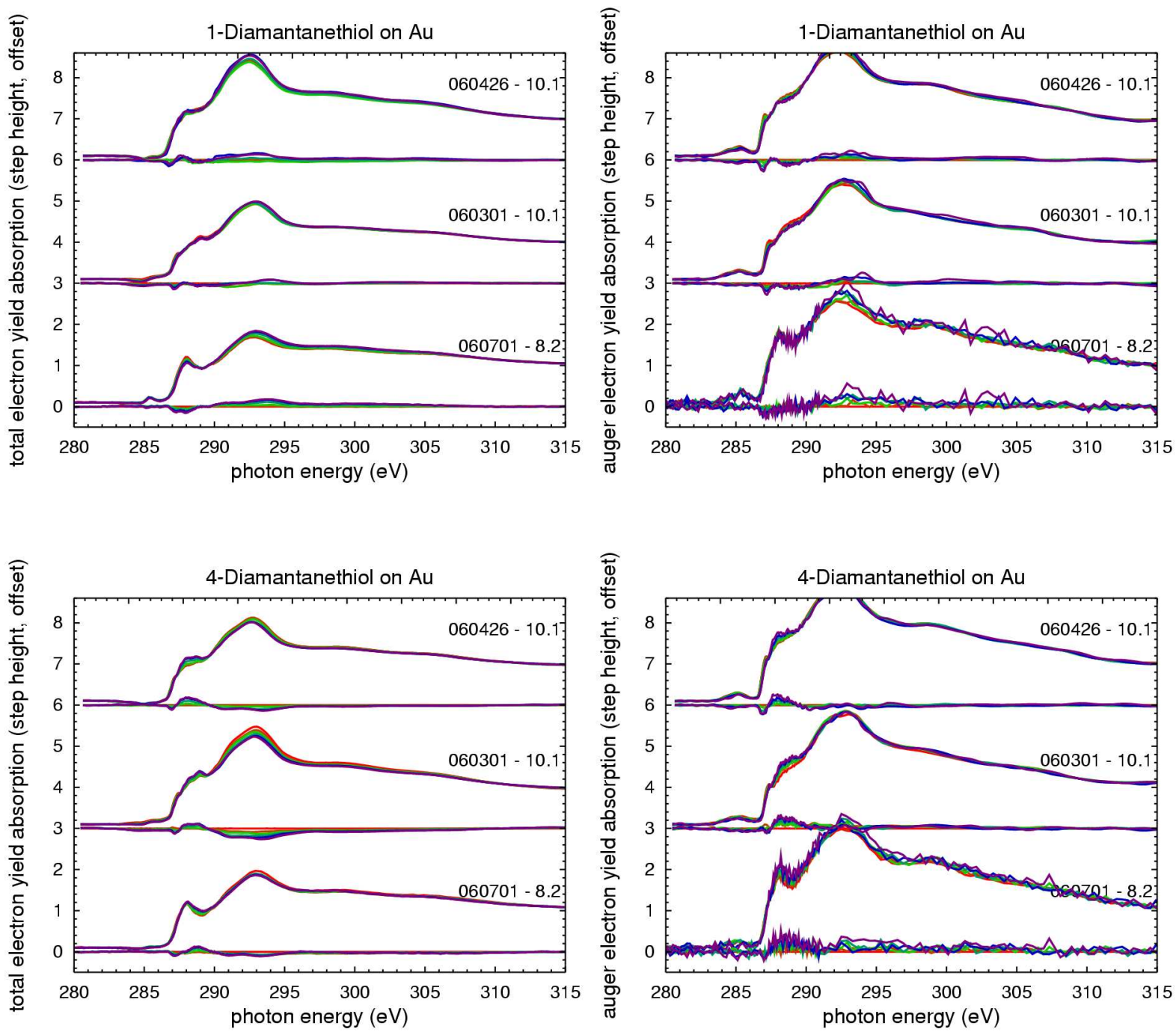


\section{Additional NEXAFS Data}

Total electron yield appears on the left, while carbon Auger yield appears on the right.
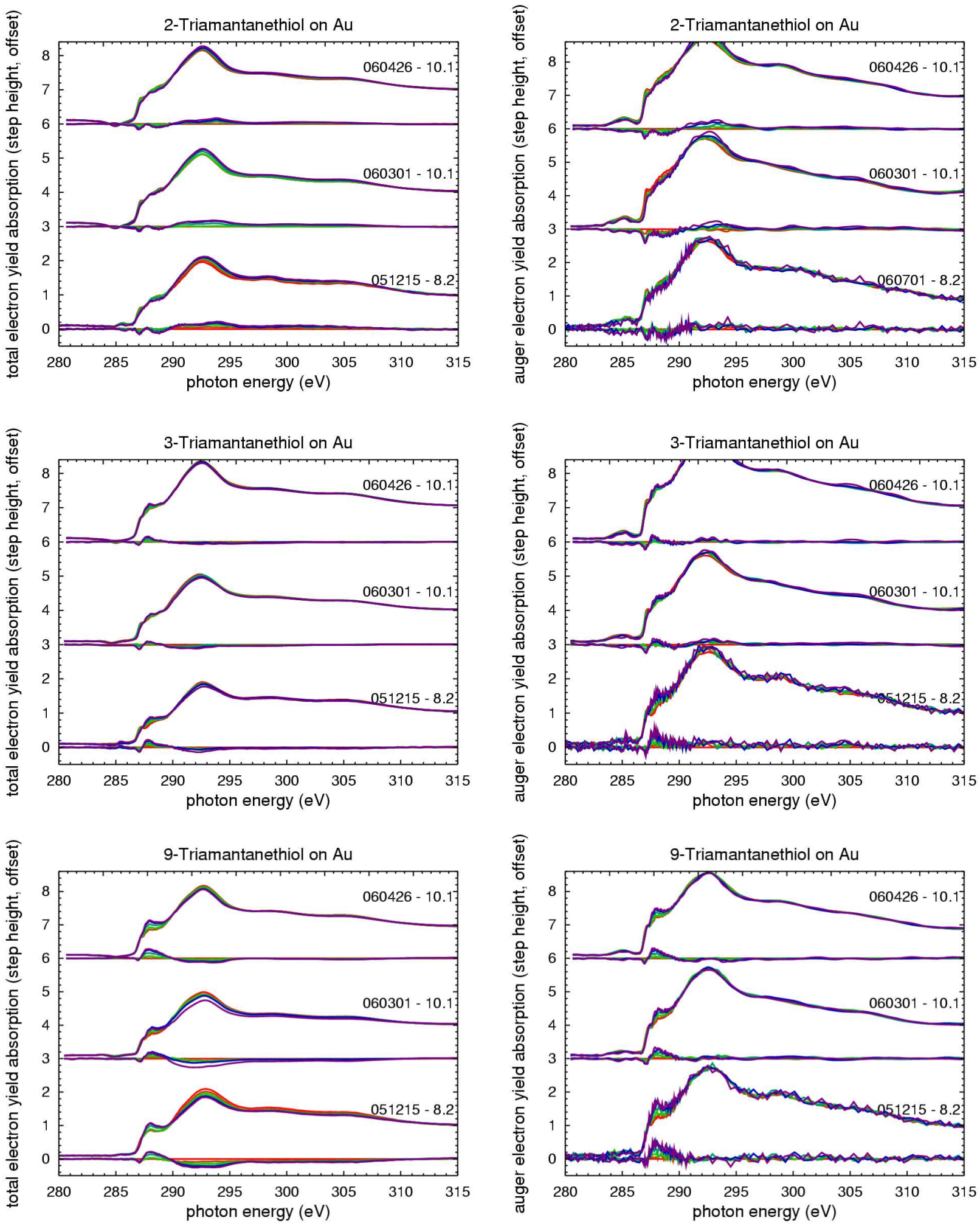


\section{Additional NEXAFS Data}

Total electron yield appears on the left, while carbon auger yield appears on the right.
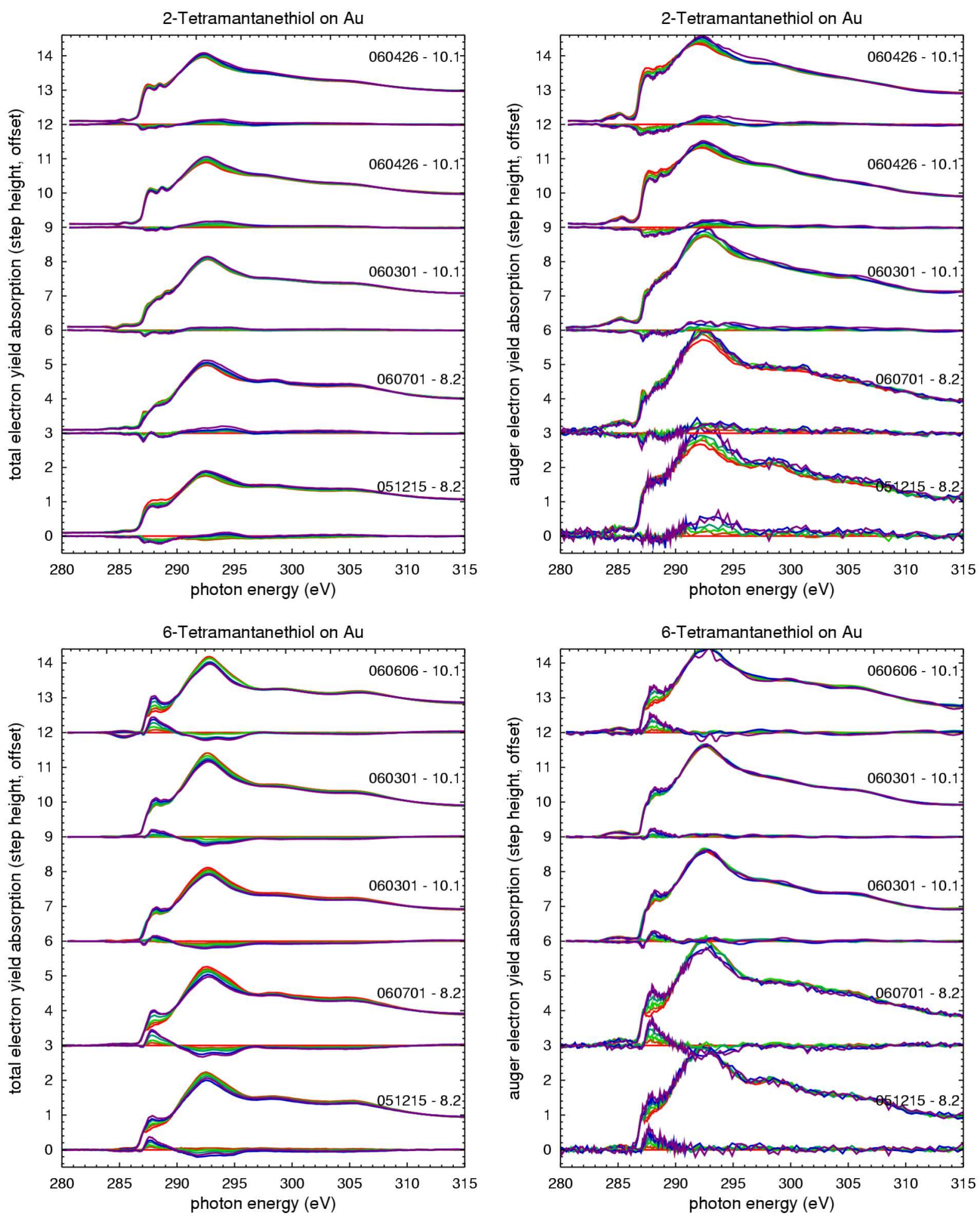


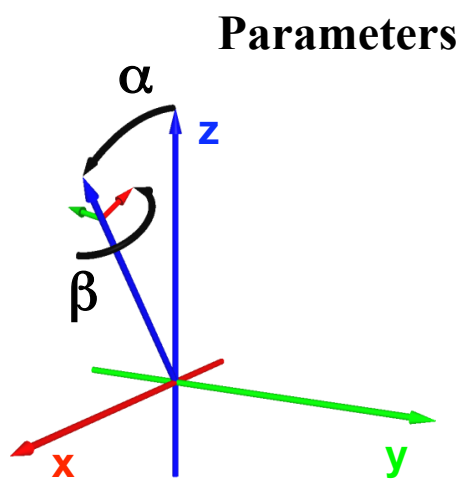

In Equation 3 in the text, intensity of the NEXAFS resonance is given as a function of alpha and beta of a molecular axis. Alpha is the tilt or polar angle, and beta is the twist or dihedral angle. These angles are depicted in this figure.

A molecular axis is chosen; in this work, two convenient axes are used. The first is along the [110] direction aligned with the long axis of the molecules diamantane and larger. This is a common axis along the rod-like diamondoids in this work, similar to the axis chosen for alkanethiols. The second is an axis chosen along the S-C bond in each molecule. The NEXAFS tilt/twist plots in the text and supplemental information are presented with respect to the molecular axis. These axes for all diamondoid thiols are presented, with $\mathrm{x}, \mathrm{y}$, and $\mathrm{z}$ molecular axes depicted in red, green, and blue respectively.

\section{Molecular axis defined along the [110] direction}

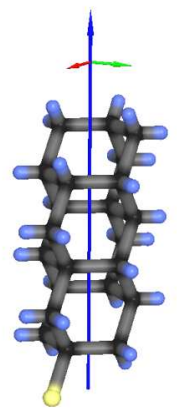

Tetramantane-6-thiol (structure 8)

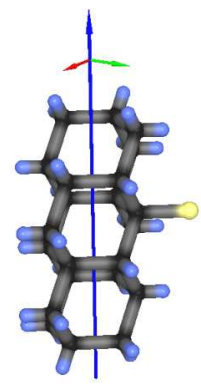

Tetramantane-2-thiol (structure 7)

\section{Molecular axis defined along the S-C bond}
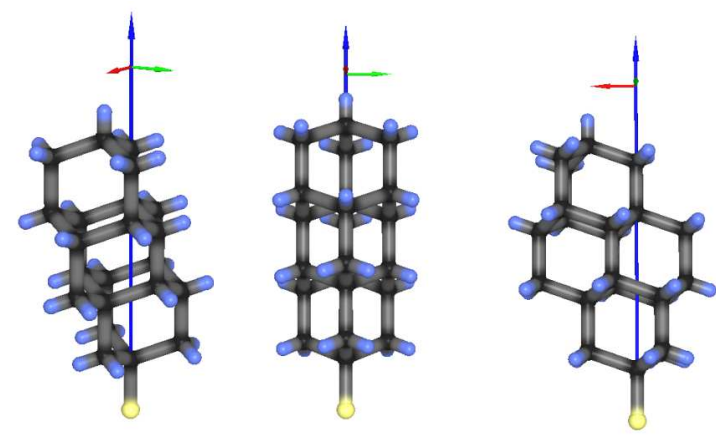

Tetramantane-6-thiol (structure 8) Shown looking down the $\mathrm{x}$-axis (middle) and y-axis (right)
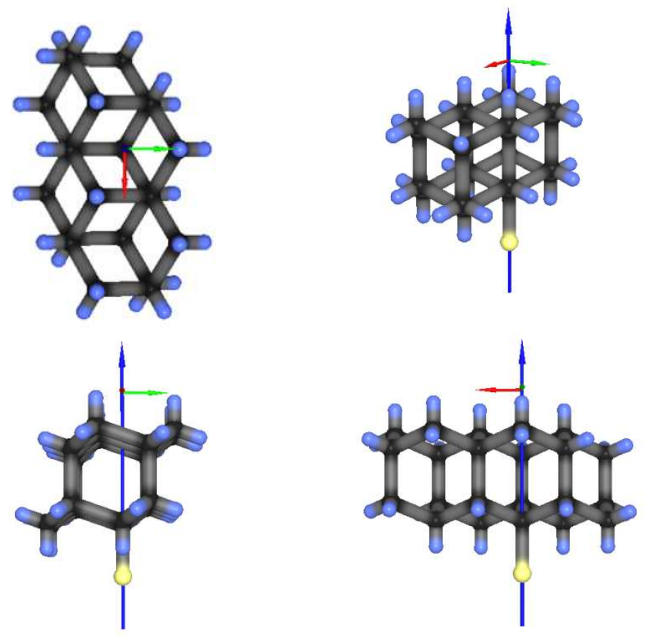

Tetramantane-2-thiol (structure 7) Various views of the same molecular axis are depicted. 


\section{Molecular Axes to Describe Orientation}

The balance of the molecular axes used for the diamondoidthiols in this work. For simplicity, as the general consensus in the literature points towards cleavage of the S-H thiol bond upon absorption, only the $\mathrm{S}$ atom is shown.

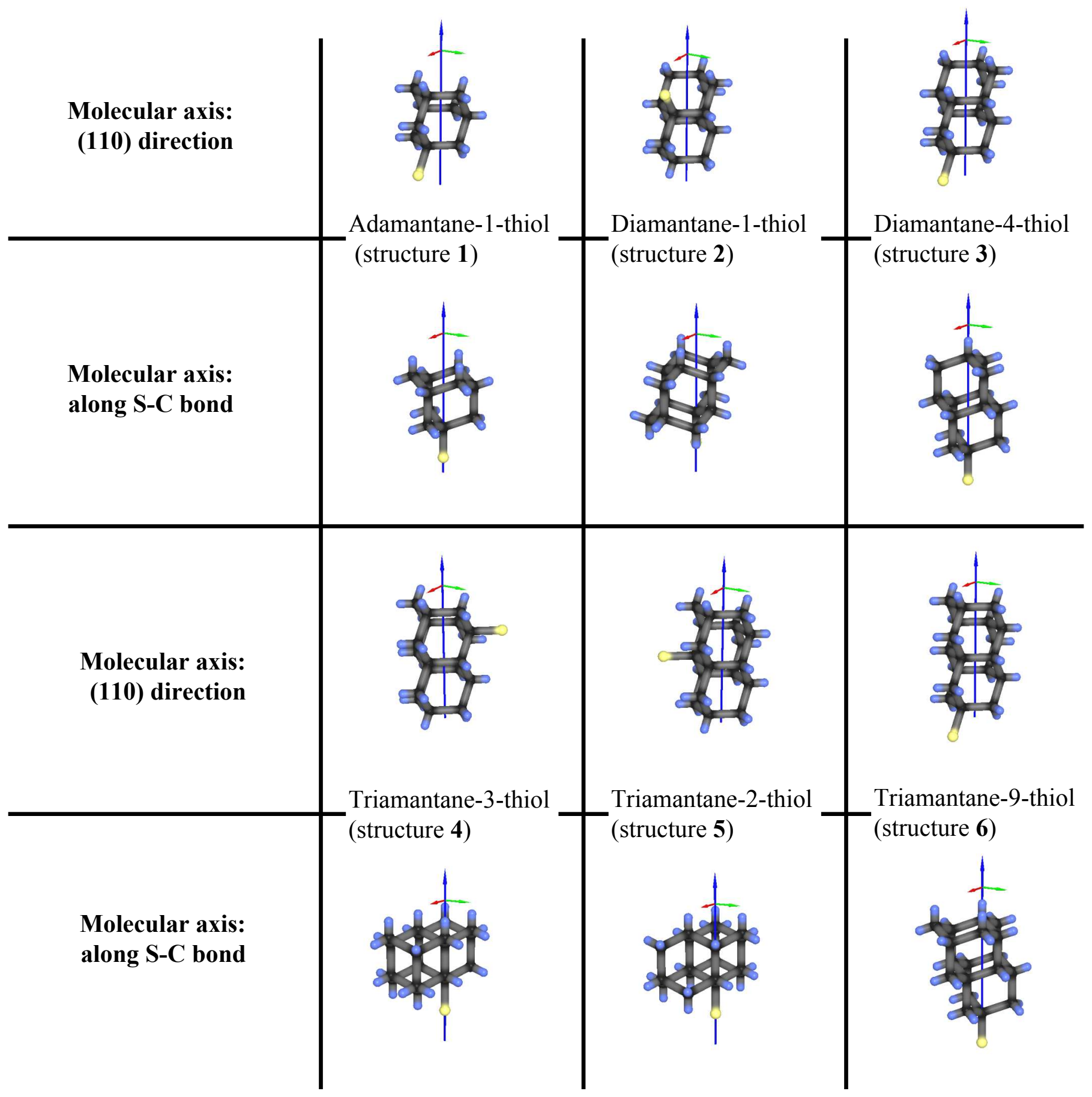




\section{Additional Derived Orientations}

The two best datasets for each sample were used to derive orientations. Each of these are plotted on the following pages. The color scheme is as follows: purple - sterically impossible; blue, sterically possible; green shades: $\mathrm{C}-\mathrm{C} \sigma^{*}$ or $\mathrm{C}-\mathrm{H} \sigma^{*}$ within $95 \%$ confidence limits of linear regression of the ratios of intensities as described in the text; orange: overlap between $\mathrm{C}-\mathrm{H} \sigma^{*}, \mathrm{C}-\mathrm{C} \sigma^{*}$, and white: $\mathrm{C}-\mathrm{H} \sigma^{*}, \mathrm{C}-\mathrm{C} \sigma^{*}$ and sterically possible all overlap.

First, the tilt/twist plots for [121]tetramantane-6-thiol (structure 8 in the text)

Molecular axis defined along the [110] direction

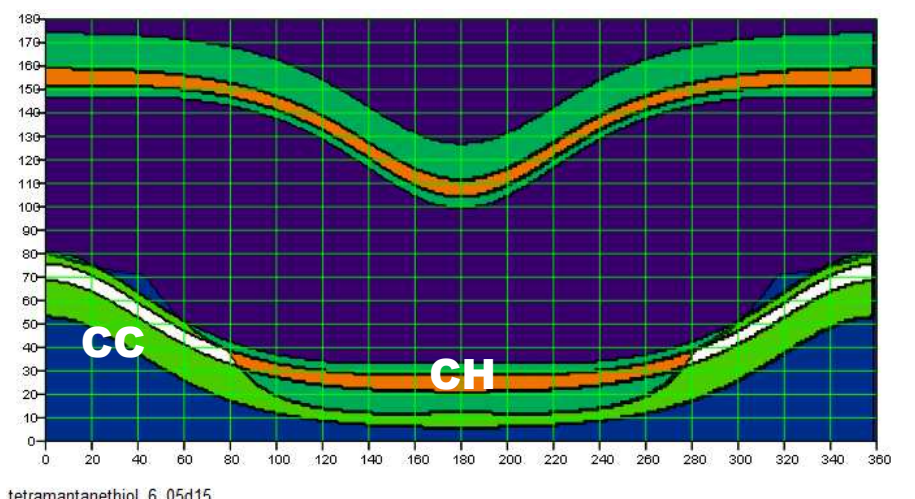

tetramantanethiol_6_05d15

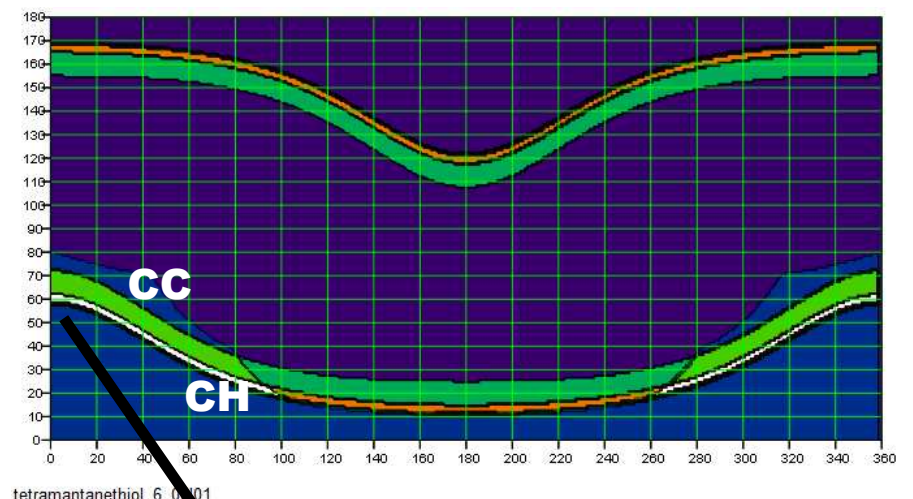

tetramantanethiol_
Molecular axis defined along S-C bond

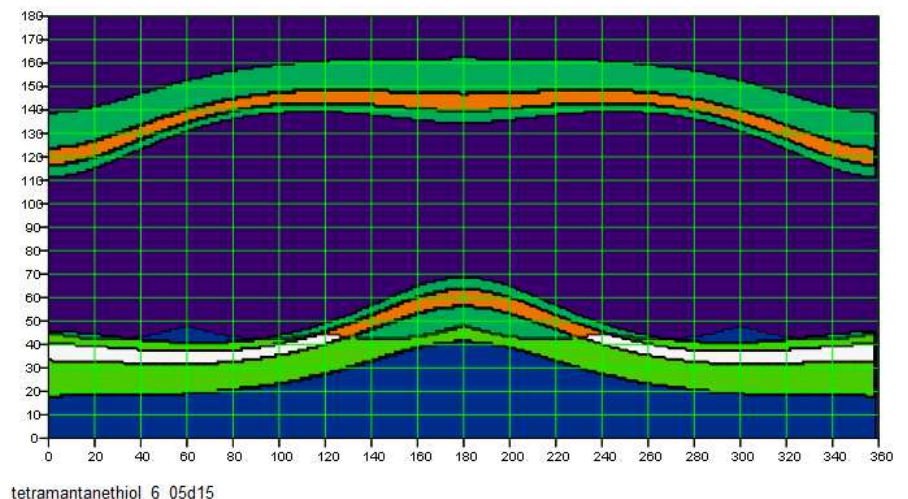

tetramantanethiol 6 _05d15

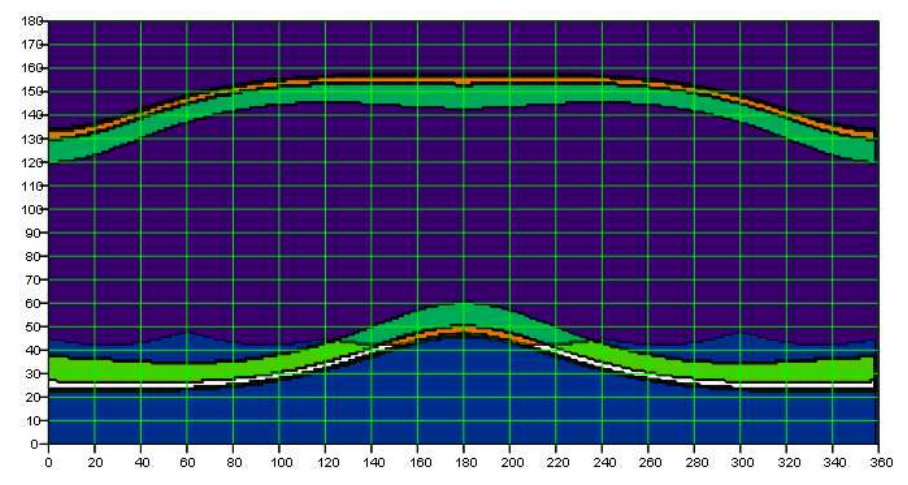

tetramantanethiol_6_06101

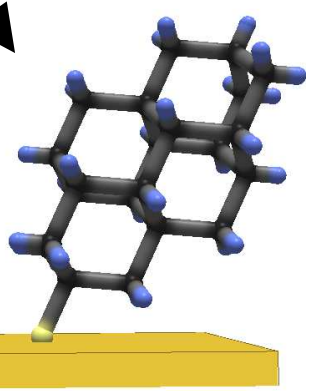

The upper and lower datasets are consistent; in the right panes (molecular axis defined along the $\mathrm{S}-\mathrm{C}$ bond) the $\mathrm{C}-\mathrm{H}$ resonace returns $36.5+/-4$ (upper sample) and $26+/-3$ degrees (lower sample). We thus estimate, away from the sterically impossible, a polar angle for $30+/-10$ for the S-C bond.

At left, a side view of one of these orientations (polar angle of 60 degrees, dihedral angle of 0 degrees) is depicted. 
[121]tetramantane-2-thiol (structure 7 in the text)

Molecular axis defined along the [110] direction

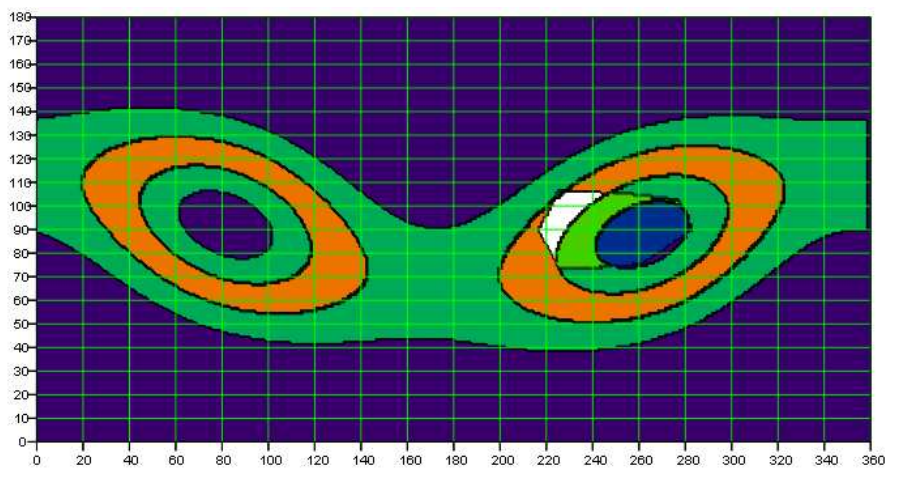

tetramantanethiol_2_05d15

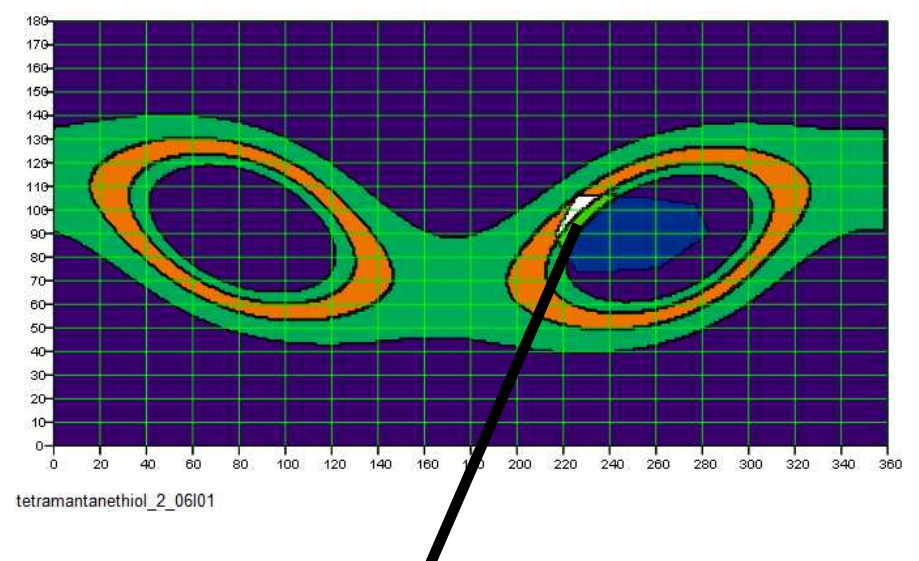

\section{Molecular axis defined along S-C bond}

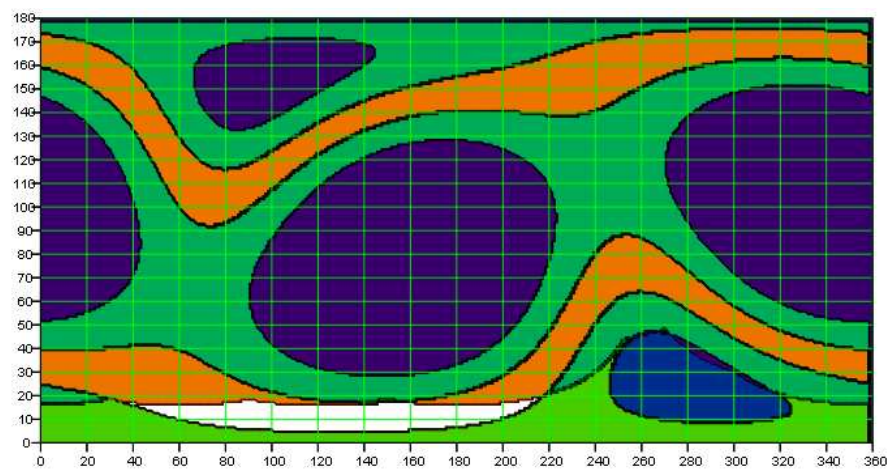

tetramantanethiol_2_05d15

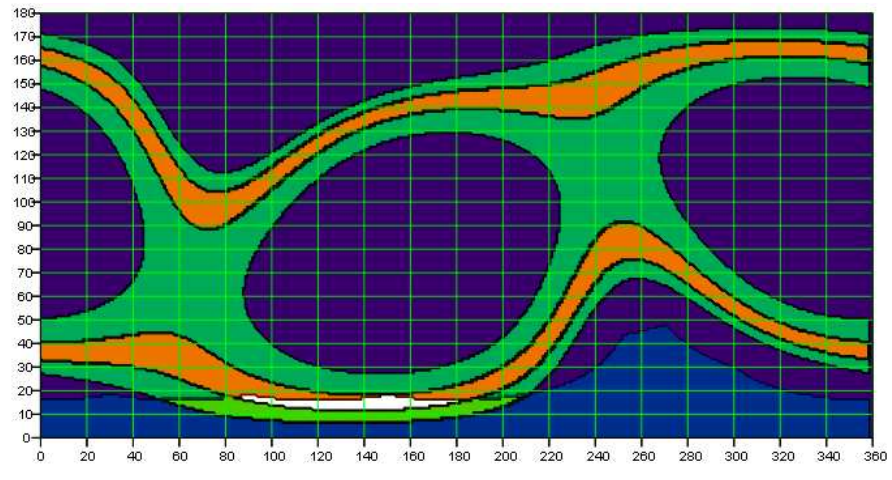

tetramantanethiol_2_06101

In this case, with the thiol positioned at the side of the molecule, in the left hand panes, both show that the long axis lies between 80 and 105 degrees in the top pane, and between 90-105 in the lower pane, a very prostrate position. When the molecular axis is defined along the S-C bond, the NEXAFS consistently does not overlap with the sterically accessible twist about the long axis which would allow a lowerenergy, canted orientation of the S-C bond. Instead, The NEXAFS overlaps the sterically possible in a region where the polar angle of the $\mathrm{S}-\mathrm{C}$ bond is expected to be lower than about 15 degrees.

The model at left of tetramantane-2-thiolate on gold at about 95 degrees polar angle, dihedral angle 230 degrees, using the long-axis as the molecular axis (as in the left panes above.) 
Triamantane-9-thiol (structure 6)

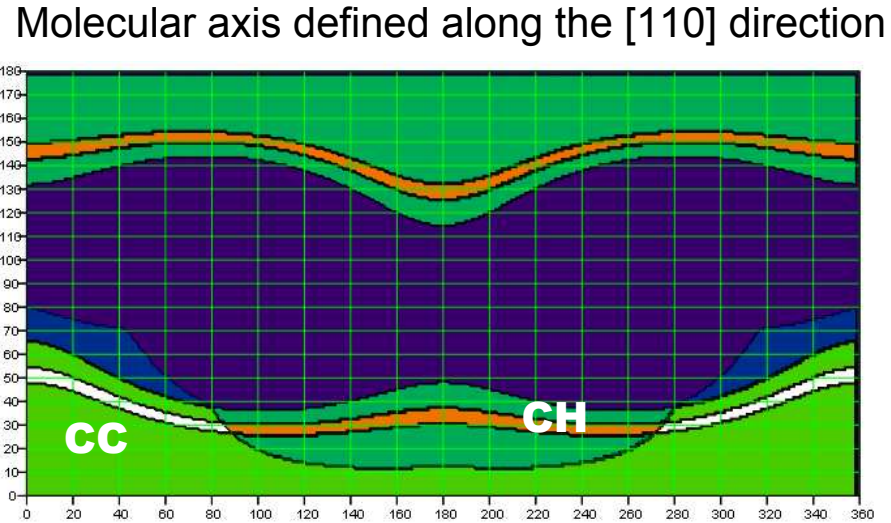

triamantanethiol_9_05d15

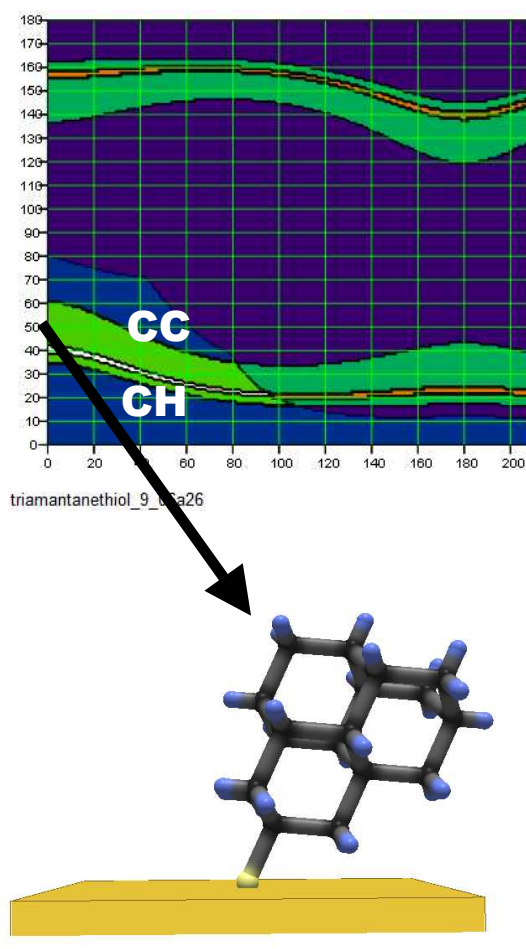

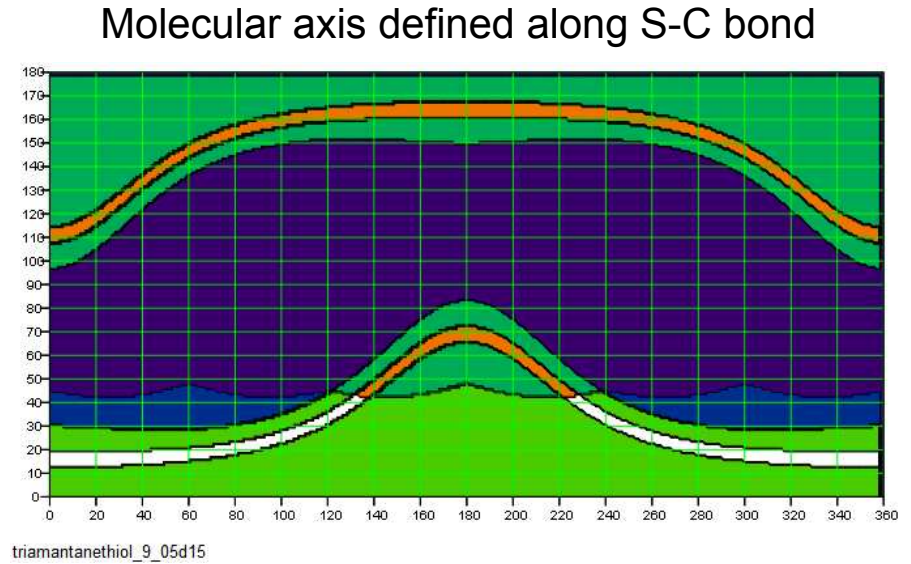

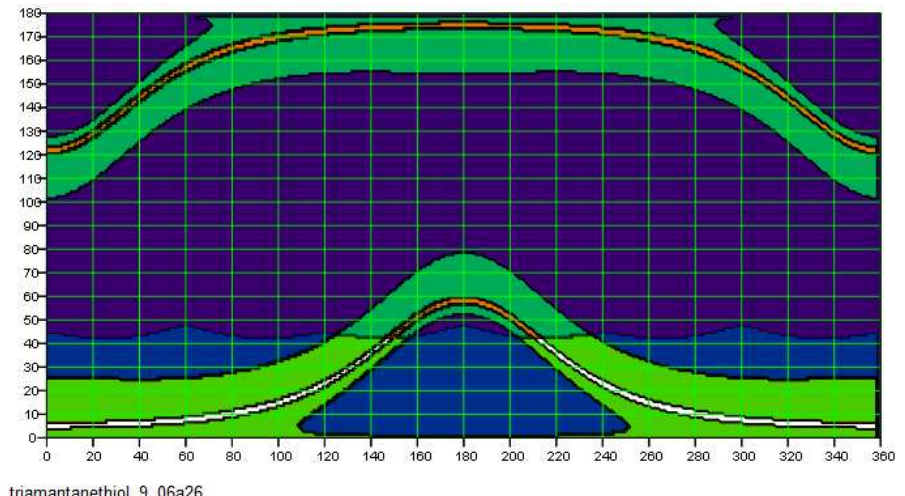

triamantanethiol_9_06a26

The systematic errors in the NEXAFS angular dependence are becoming more obvious. The quantitative analysis can be performed, but at best, a qualitative picture is obtained. In the sample represented in the top panes, the $\mathrm{C}-\mathrm{C} \sigma^{*}$ resonances allow for nearly any orientation where the S-C bond polar angle is smaller than 30 degrees, and any sterically possible orientation of the molecule with a twist of $180+/-60$ degrees. The $\mathrm{C}-\mathrm{H}$ resonance returns, for the polar angle of the S-C bond, roughly $15+/-5$ degrees for $+/-90$ degrees twist, and then approaches the sterically limited orientations as the dihedral angle increases. The lower panes show a C-C resonance where the polar angle of the $\mathrm{S}-\mathrm{C}$ bond is between 5 and about 27 degrees; the $\mathrm{C}$ $\mathrm{H} \sigma^{*}$ returns a more normal polar angle. (In this case, the $\mathrm{C}-\mathrm{H} \sigma^{*}$ did not completely overlap with the $\mathrm{C}-\mathrm{C} \sigma^{*}$.) The data are consistent with an upright but canted orientation of the triamantane-9-thiolate molecules on the gold surface. 
Triamantane-2-thiol (structure 5)

Molecular axis defined along the [110] direction

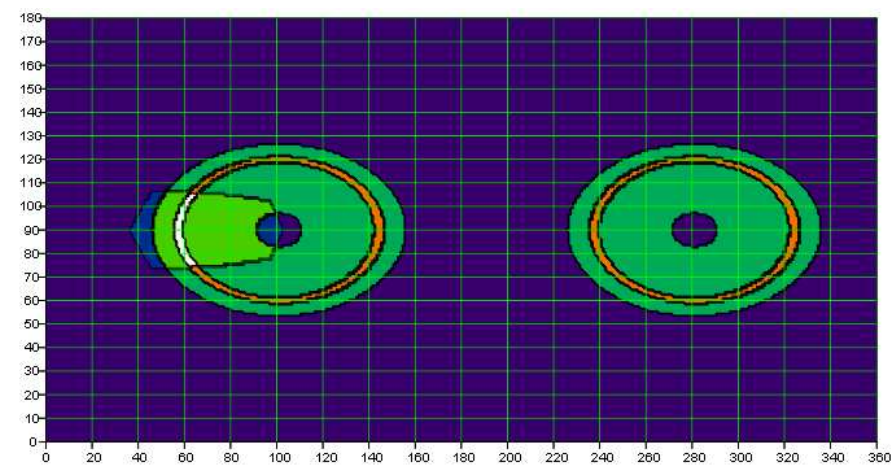

triamantanethiol_2_06101

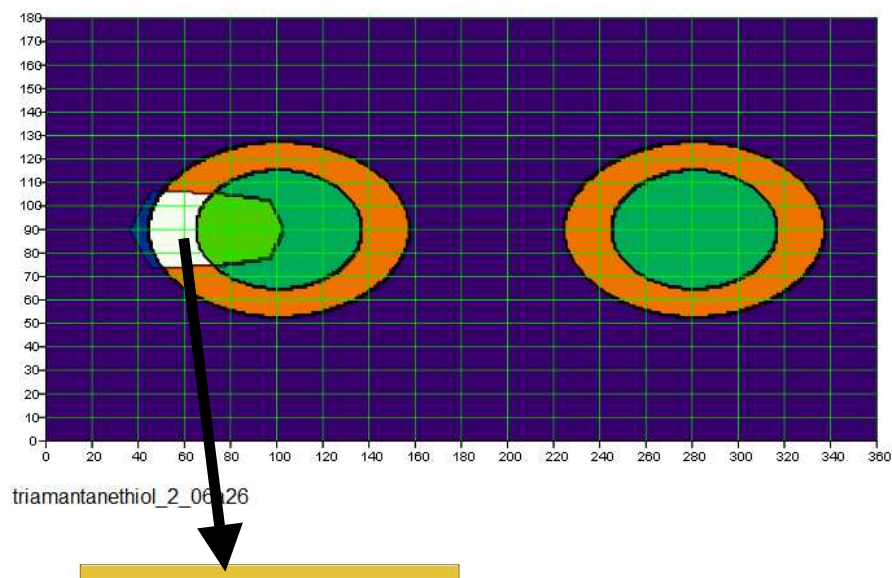

Molecular axis defined along S-C bond

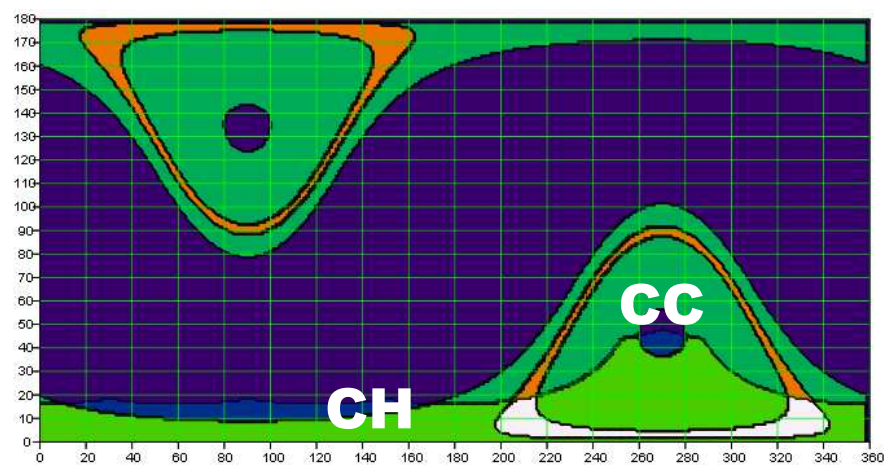

triamantanethiol_2_06a26

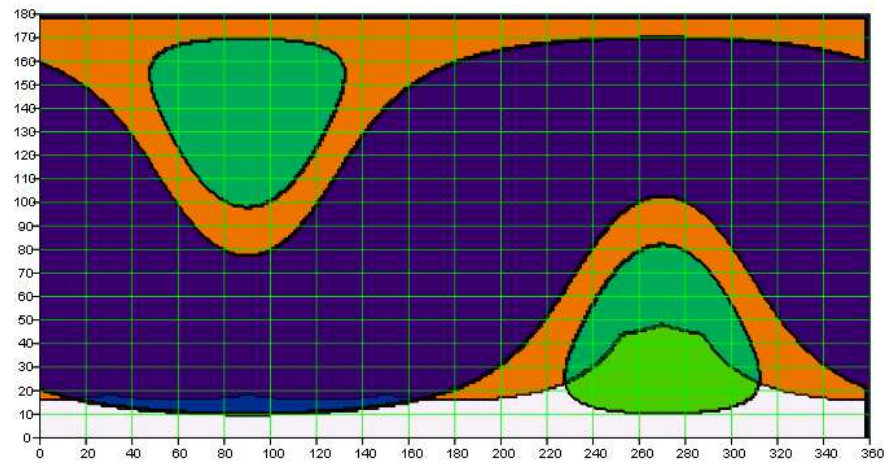

triamantanethiol_2_06a26

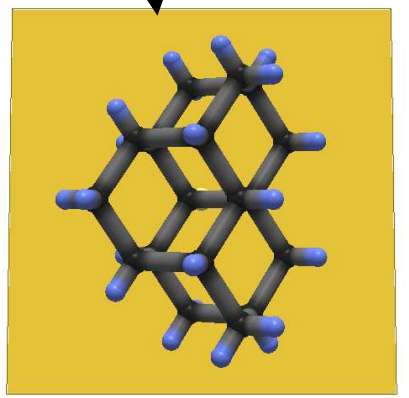

The triamantane-2-thiol was the most difficult to dissolve. Although the materials were stored in small closed vials, fresh material appeared to dissolve more readily in small amounts of toluene that was then diluted to $\sim 1 \mathrm{mmol}$ with ethanol. Both sets of $\mathrm{C}-\mathrm{H} \sigma^{*}$ data are very similar, supporting a normal orientation (less than 15 degrees) of the $\mathrm{S}-\mathrm{C}$ bond. The $\mathrm{C}-\mathrm{C} \sigma^{*}$ data in the lower panes has very large errors and overlaps most of the sterically accessible orientations. 
Triamantane-3-thiol (structure 4)

Molecular axis defined along the [110] direction

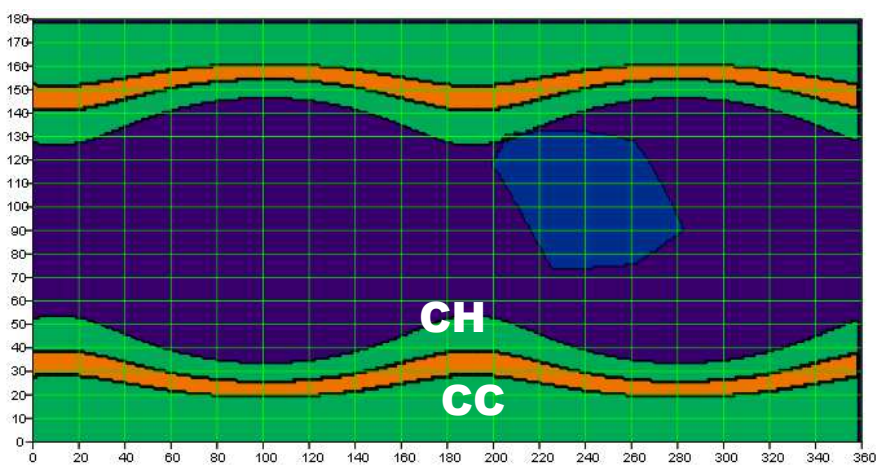

triamantanethiol_3_05d15

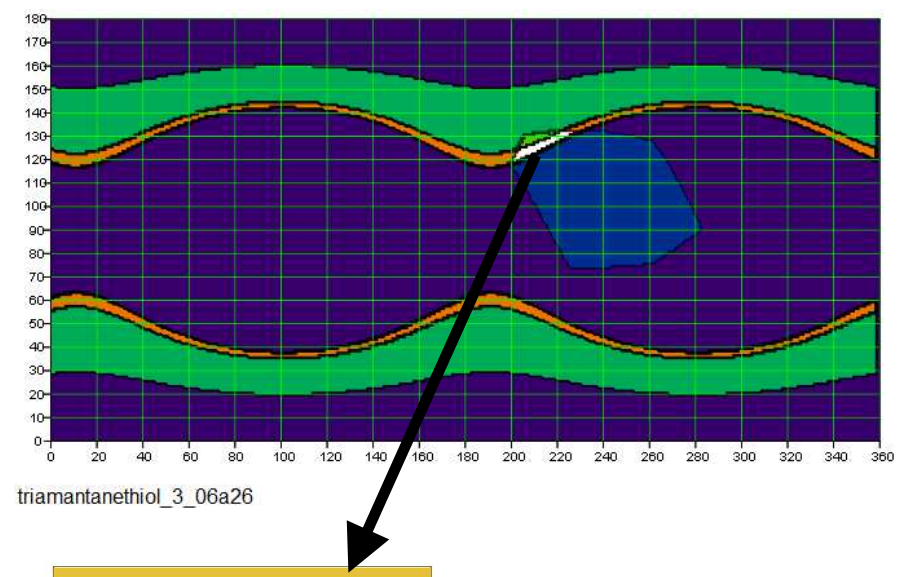

Molecular axis defined along S-C bond

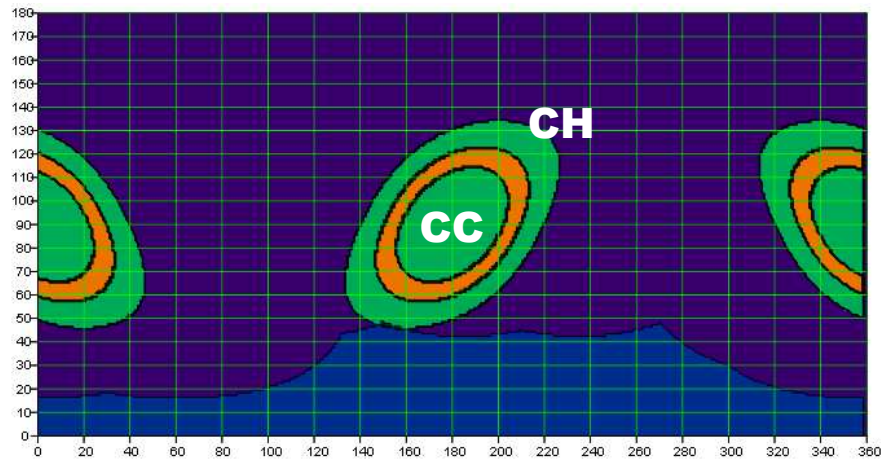

triamantanethiol_3_05d15

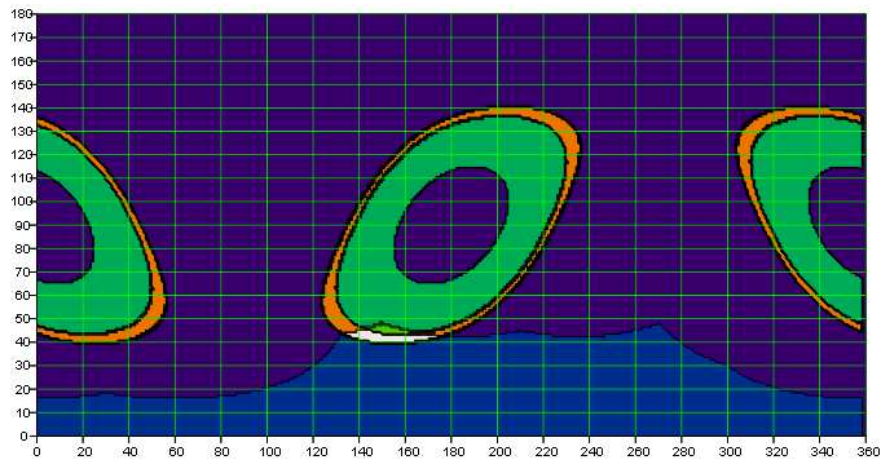

triamantanethiol_3_06a26

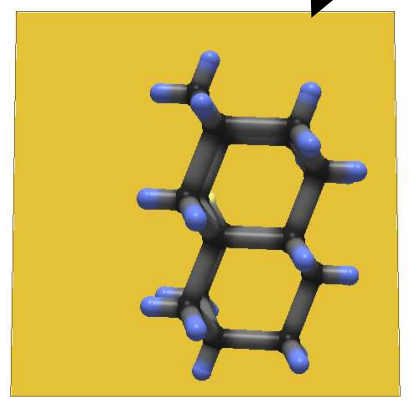

In the top case, the C-C $\sigma^{*}$ doesn't overlap with sterically possible orientations of the molecule at all. The $\mathrm{C}-\mathrm{H} \sigma^{*}$ resonances come close. In the lower panes, both resonances barely overlap. A molecule modeled at an orientation near the area of overlap shown exhibits an orientation that appears to present a [110] surface. 
Diamantane-4-thiol (structure 3)

Molecular axis defined along the [110] direction

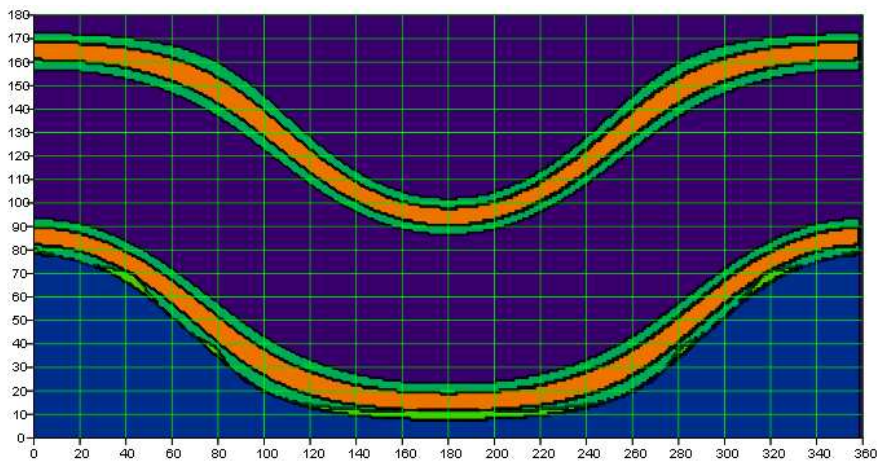

diamantanethiol_4_05d15

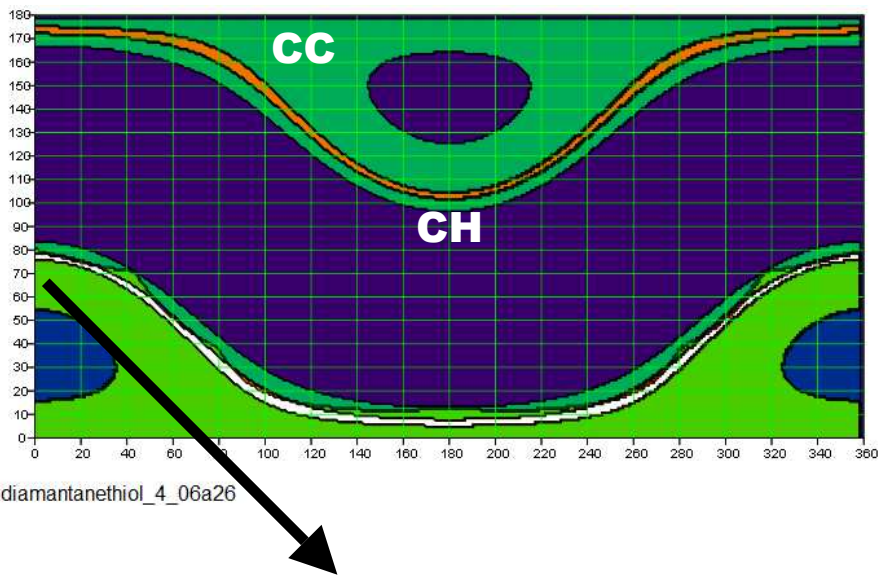

Molecular axis defined along S-C bond

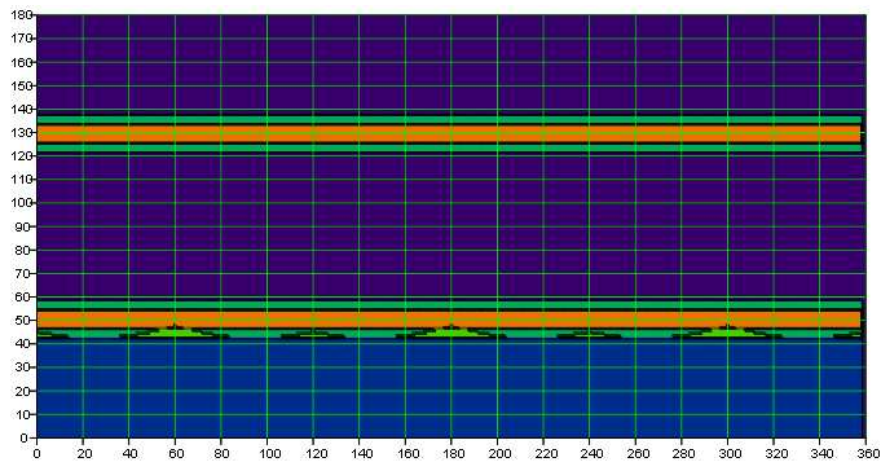

diamantanethiol_4_05d15

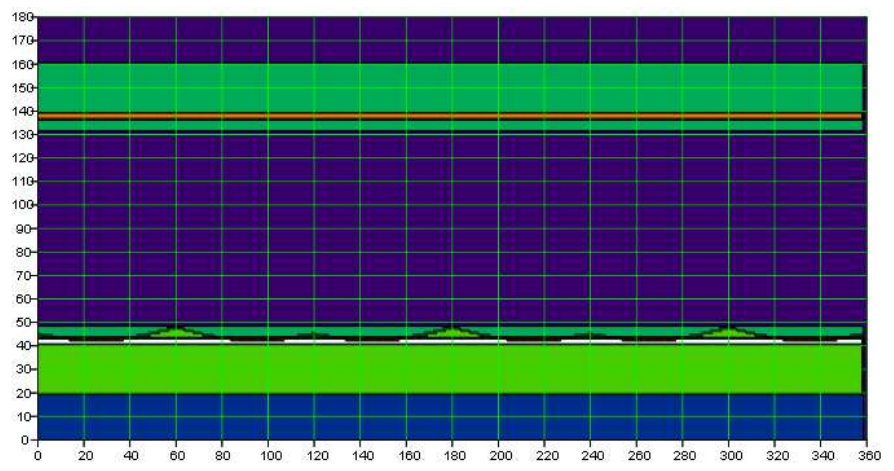

diamantanethiol_4_06a26

In diamantane-4-thiol, both sets of data for the $\mathrm{C}-\mathrm{H} \sigma^{*}$ resonance show a canted orientation that is very near the steric limit. The same is true for the $\mathrm{C}-\mathrm{C} \sigma^{*}$ resonance in the sample prestented in the upper panes. In the second sample (lower panes), the $\mathrm{C}-\mathrm{C} \sigma^{*}$ resonance has larger errors, and returns a $\mathrm{C}-\mathrm{S}$ polar angle of about $35+/-15$ degrees. 
Diamantane-1-thiol (structure 2)

Molecular axis defined along the [110] direction

Molecular axis defined along S-C bond
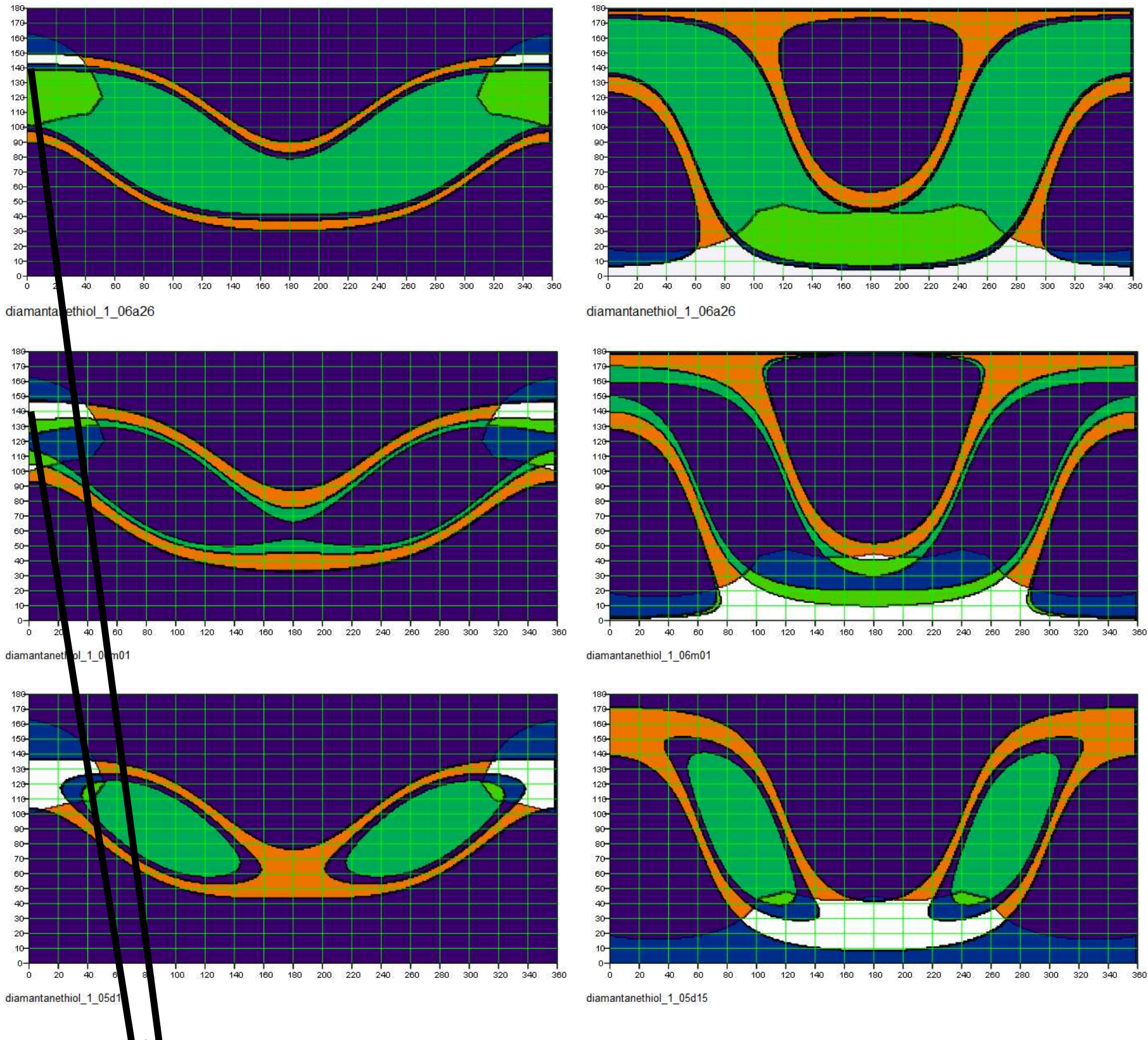

diamantanethiol_1_06a26

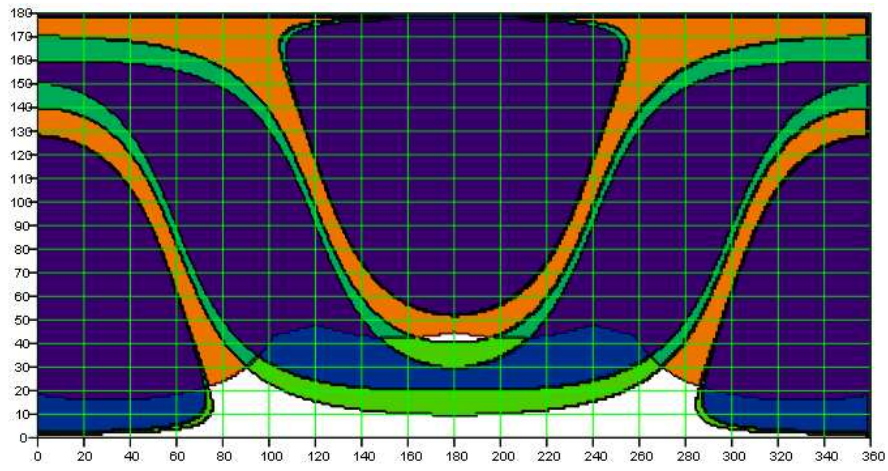

diamantanethiol_1_06m01

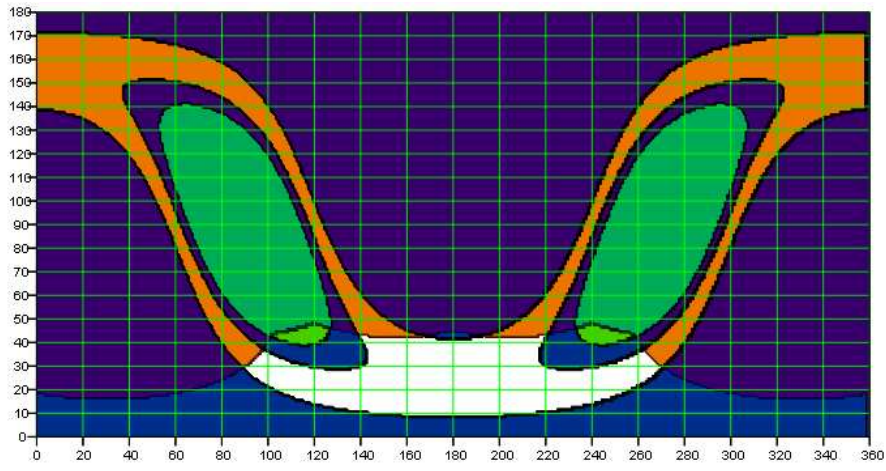

diamantanethiol $105 \mathrm{~d} 15$

Three samples are shown to illustrate that systematic errors are becoming more apparent. In the best two datasets (the top two panes) the more dependable $\mathrm{C}-\mathrm{H} \sigma^{*}$ resonances indicate a normal orientation of the C-S bond, consistent with the S-C bond in tetramantane-2-thiol and triamantane-2-thiol, but the third best dataset returns a canted orientation for the S-C bond. Since the resonances do not overlap in the top and bottom panes, the $\mathrm{C}-\mathrm{H} \sigma^{*}$ are presented in orange and white. 
Adamantanethiol (structure 1)

Molecular axis defined along the [110] direction

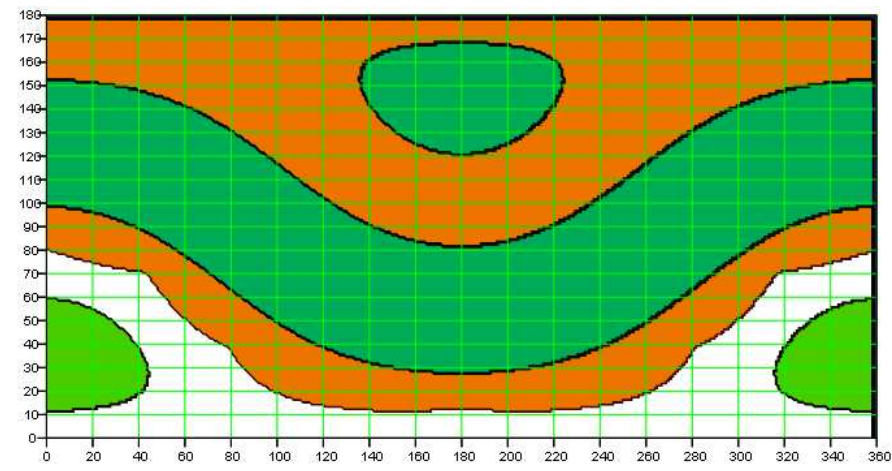

adamantanethiol_06a26

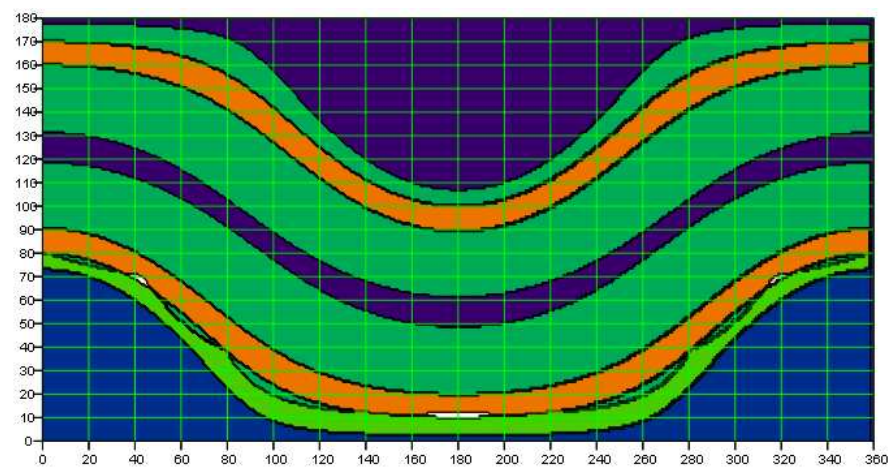

adamantanethiol_06m01
Molecular axis defined along S-C bond
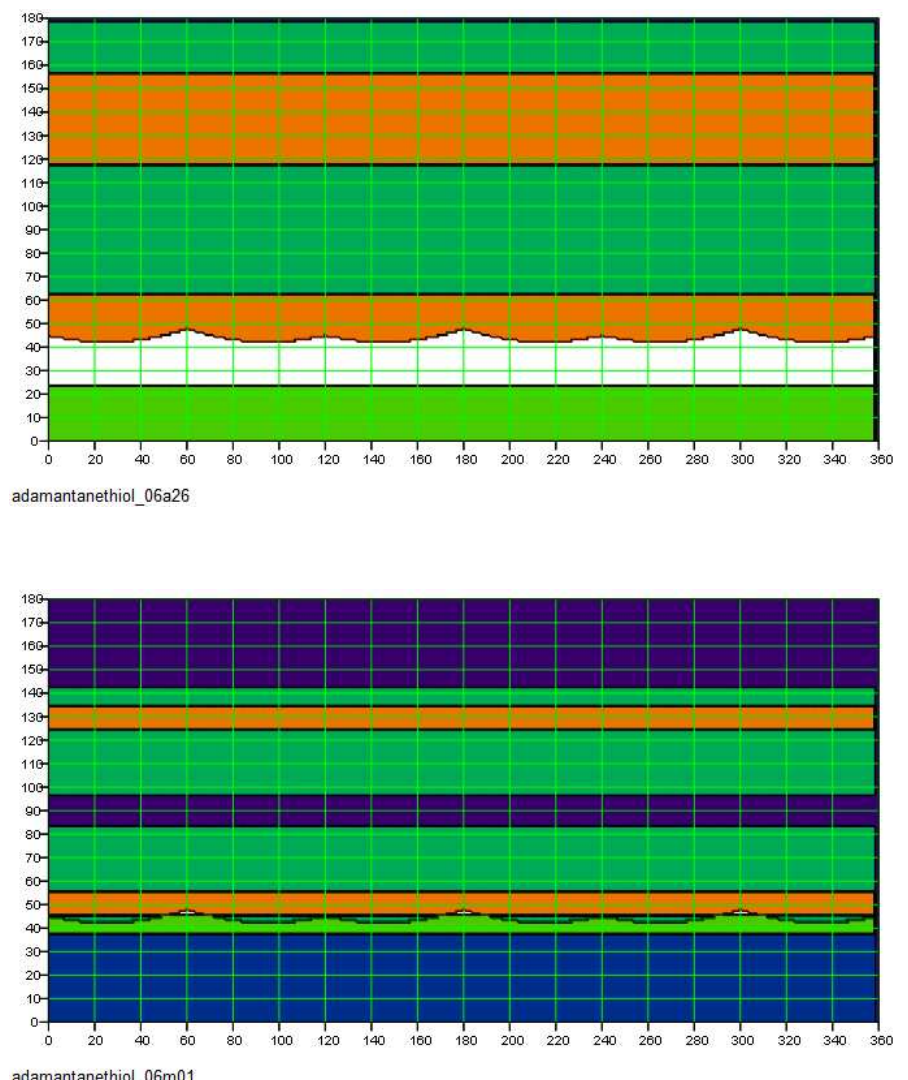

adamantanethiol $06 \mathrm{~m} 01$

Using the building block method for adamantanethiolate on gold is not particularly quantitative. As seen in the top panes, the $\mathrm{C}-\mathrm{C}$ resonance has such large errors that any combination of tilts and twists is well within the $95 \%$ confidence limits of the fits of the intensities. The fit $\mathrm{C}-\mathrm{H} \sigma^{*}$ features show a tilt (when defining the molecular axis along the S$\mathrm{C}$ bond) of greater than 25 degrees, but small systematic errors in data acquisition (i.e., carbon on the grid) or peak-fitting affect the analysis greatly. In the second sample, the errors are also large, and indicate a canted orientation that is barely sterically possible. 


\section{Computation / Data Comparison: Canted Adamantanethiolate Orientation}

Density functional theory calculations were performed on adamantanethiol using the StoBe code[S1]. Two orientations were simulated: One with an S-C bond aligned with the z-axis, simulating an S-C bond normal to the surface. The second sumulated an $\mathrm{S}-\mathrm{C}$ bond with a polar angle of 32.5 degrees.

The StoBe simulated NEXAFS:
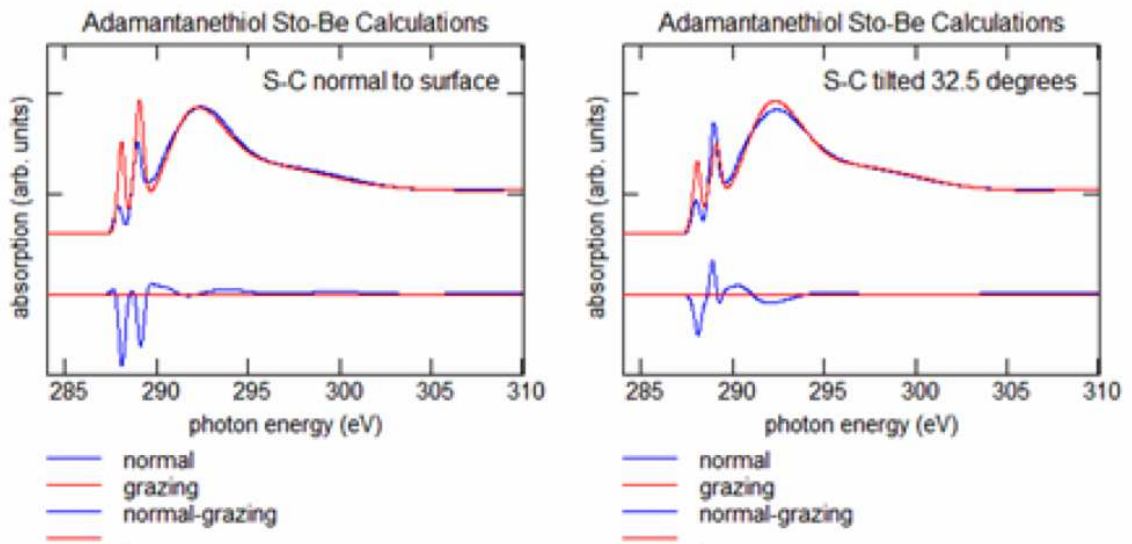

The adamantanethiolate on gold NEXAFS data:

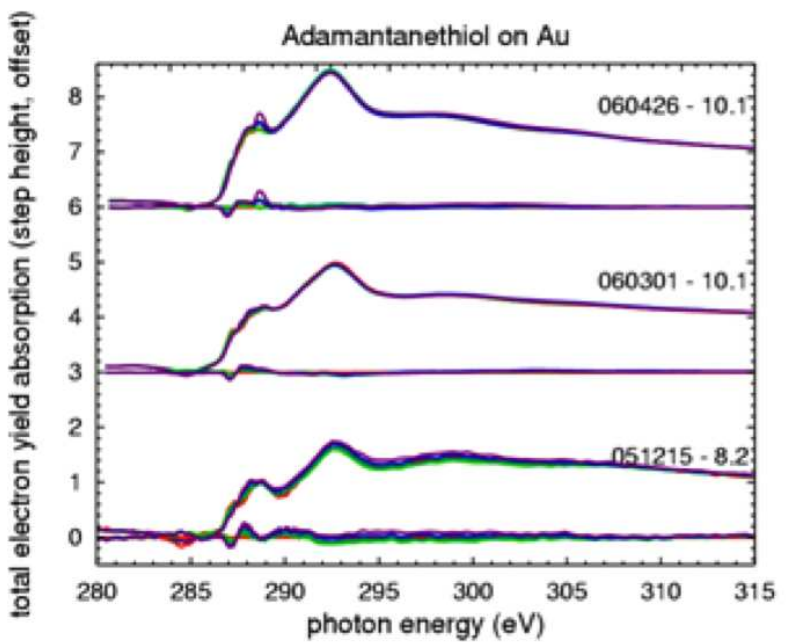

The data, especially collected on 8.2 , more closely resemble the simulation for a tilt of 32.5 degrees. This orientation is depicted below. The reader is cautioned that the calculation was performed on a single, non-interacting adamantanethiol molecule; particle-substrate and particle-particle interaction will perturb the calculated NEXAFS.

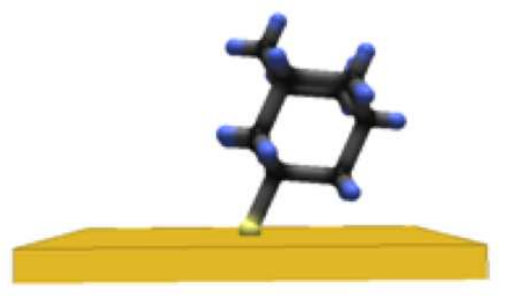




\section{Computation / Data Comparison: Angular Dependence in Tetramantanes}

In order to confirm the "upright" and "prostrate" orientation of the tetramantane molecules (structures $\mathbf{7}$ and $\mathbf{8}$ ), density functional theory calculations were performed on [121] tetramantane using the StoBe code[S1]. The resulting theoretical spectra are presented below. First, and an upright orientation was simulated by using an electric field of incident Xrays orthogonal to the [110] or long axis of the molecule for normal X-ray incidence, and an electric field along the long axis of the molecule for grazing X-ray incidence. For the simulation of a prostrate molecule the electric field was set along the thiol substitution position in 7 (perpendicular to the long axis of the molecule) for grazing incidence, and orthogonal to this direction to simulate normal incidence. Each generated spectrum represents a summation of absorption calculated for each atomic carbon center. The computed spectra exhibit clear angular dependence with the $\mathrm{C}-\mathrm{H} \sigma^{*}$ most intense at normal incidence (blue) in the upright case, and most intense at grazing incidence (red) in the prostrate case. The difference between the normal and grazing incidence spectra are presented to accentuate the angular dependent resonances. The converse is true for the $\mathrm{C}-\mathrm{C} \sigma^{*}$ region. Comparing the computed spectra to experiment on the right, the data is consistent with an upright orientation in the case of $\mathbf{8}$ on $\mathrm{Au}$, and a prostrate orientation in the case of 7 on $\mathrm{Au}(111)$.

StoBe Simulated NEXAFS, Tetramantane [121]

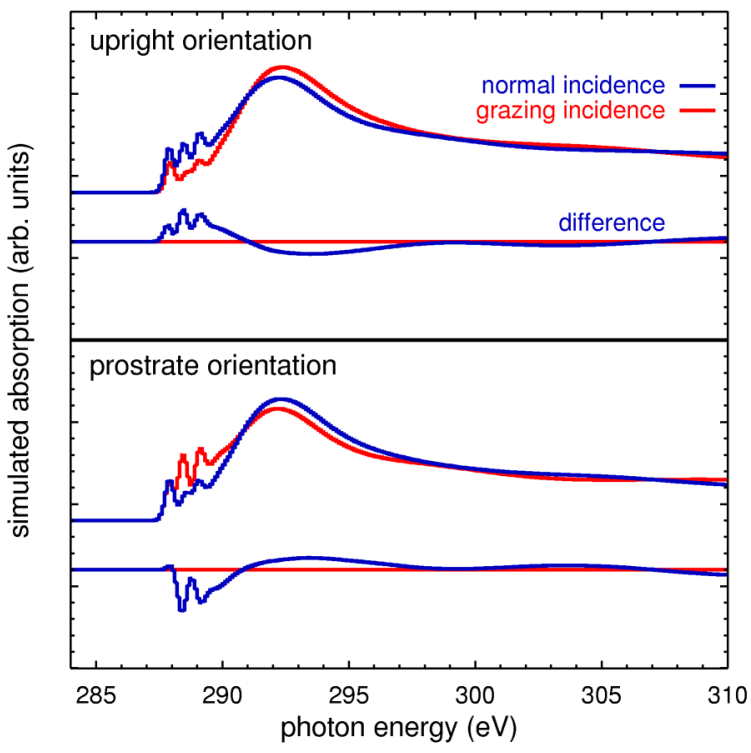

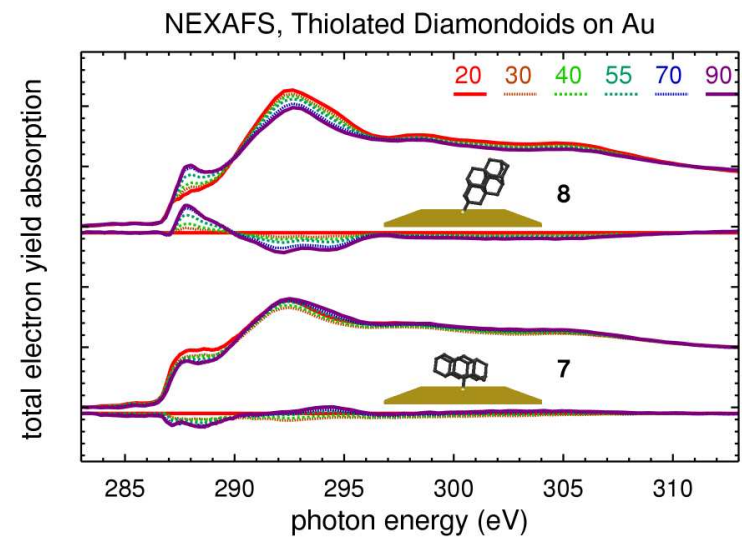




\section{Additional S 2p XPS Data: Adamantanethiolate/Au}

The S $2 p$ binding energy difference between dodecanethiolate on Au and adamantanethiolate on $\mathrm{Au}$ is presented in the text; here, the data is presented again with the $\mathrm{Au} 4 \mathrm{f}$ photoelectron peaks obtained directly before or after the $S 2 p$ scans. These Au photoelectrons were used to calibrate the binding-energy scales acquired at the synchrotron source. The data has been normalized to peak height to accentuate the small binding energy shift in the $S 2 p$, and the accuracy of the calibration using the $\mathrm{Au} 4 \mathrm{f}$ peaks. Note that here, the binding energy axis scales are the same.
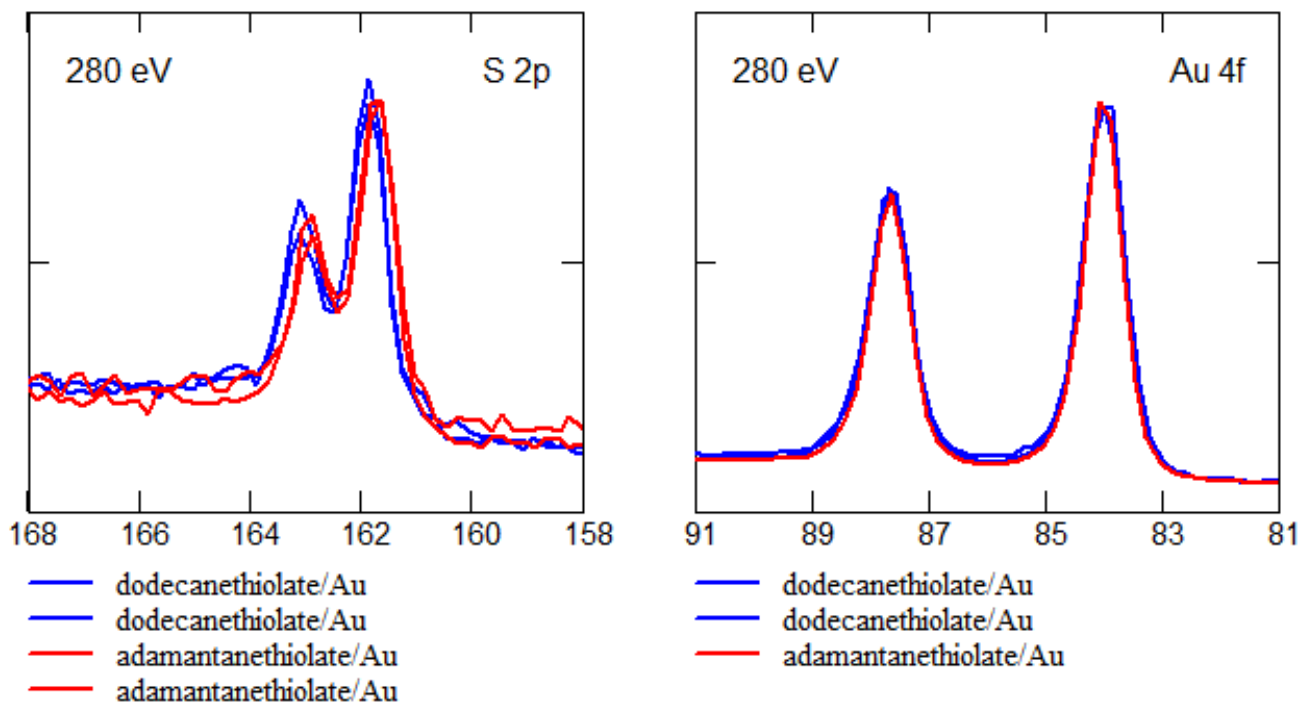

Here are an additional three samples measured at times differing by at least a few months on the two different beamlines used at SSRL. All acquisitions showed the small shift towards lower binding energy for adamantanethiolate on Au compared to dodecanethiolate adsorbed on $\mathrm{Au}$.
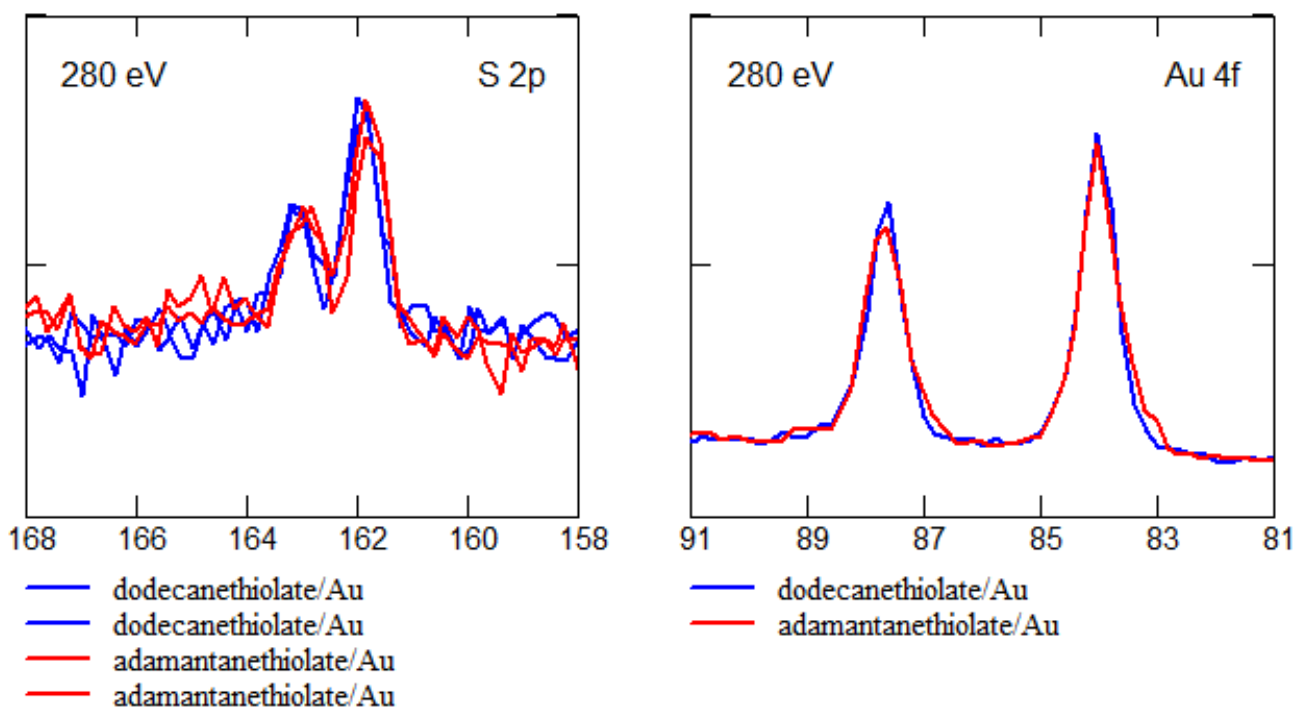
Additional S 2p XPS Data: Adamantanethiolate/Au (continued)
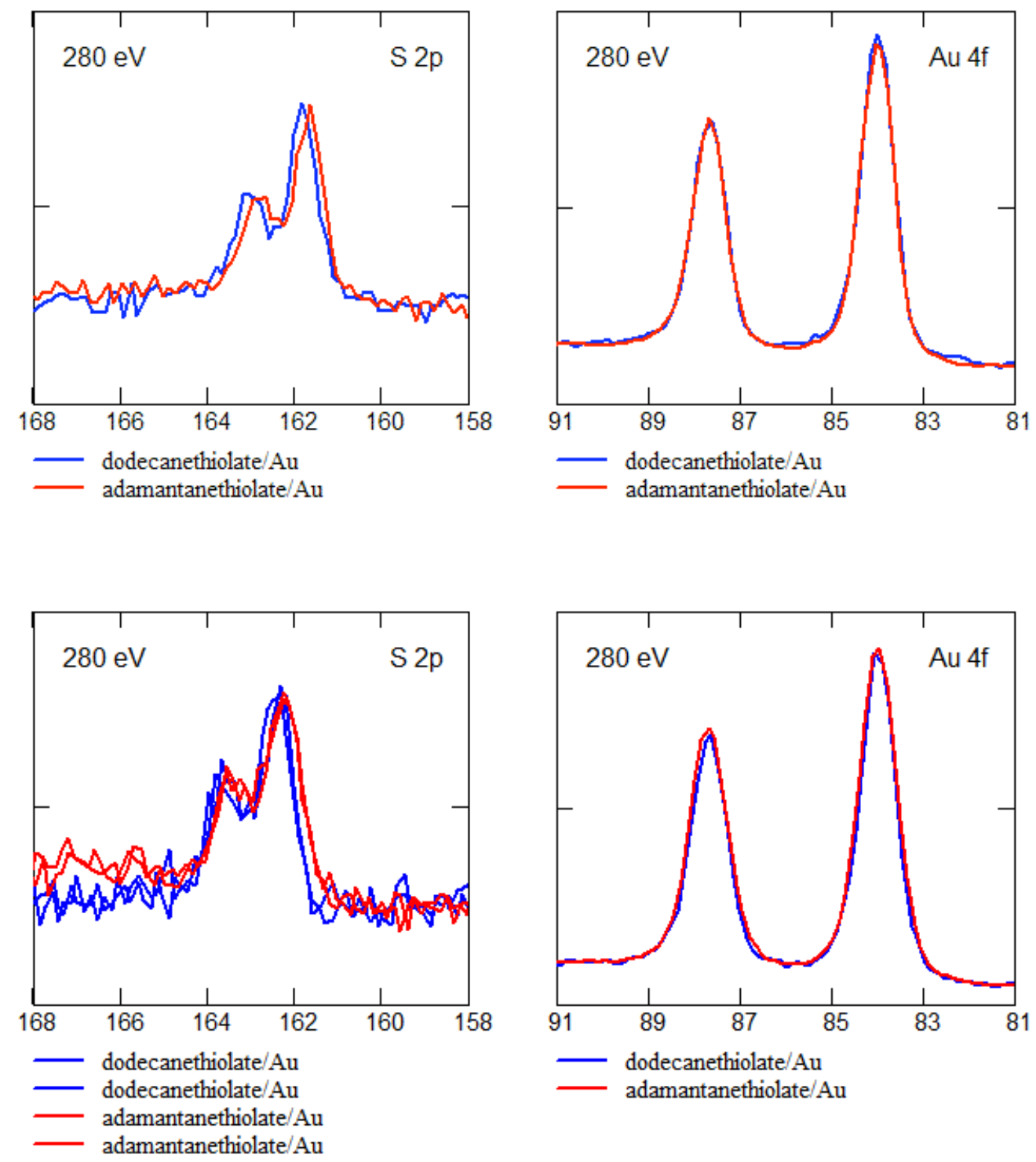

The S $2 p$ binding energy difference (in no particular order) between adamantanethiolate on $\mathrm{Au}$ and dodecanethiolate on $\mathrm{Au}$ was estimated to be: $0.15+/-0.03,0.15+/-0.03,0.19+/-$ 0.05 , and $0.15+/-0.05$. Our final estimate for the shift is $0.16+/-0.04 \mathrm{eV}$. 
Selected S 2p data from various diamondoidthiols are now presented.
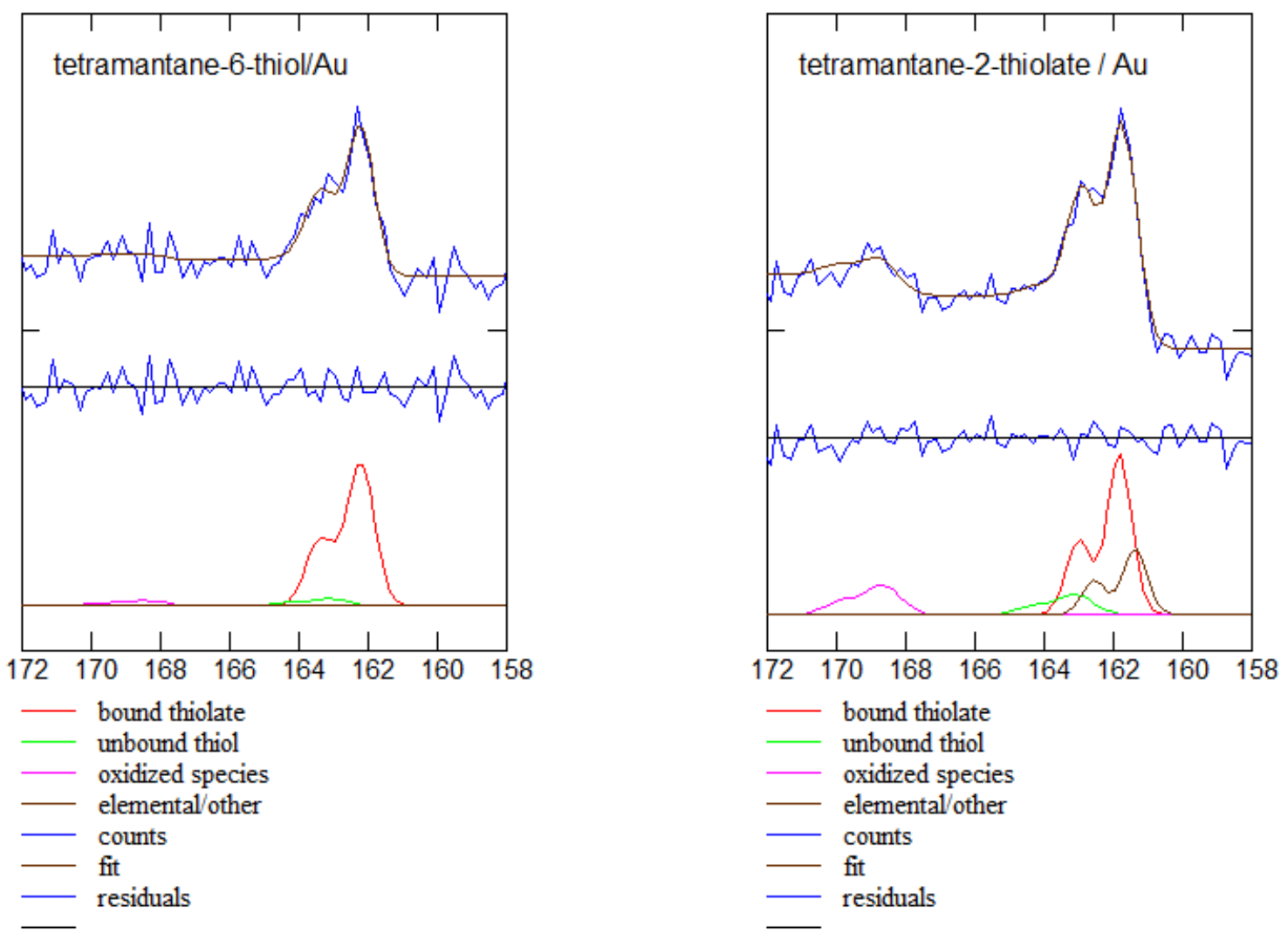

tetramantanethiolates / $\mathrm{Au}$

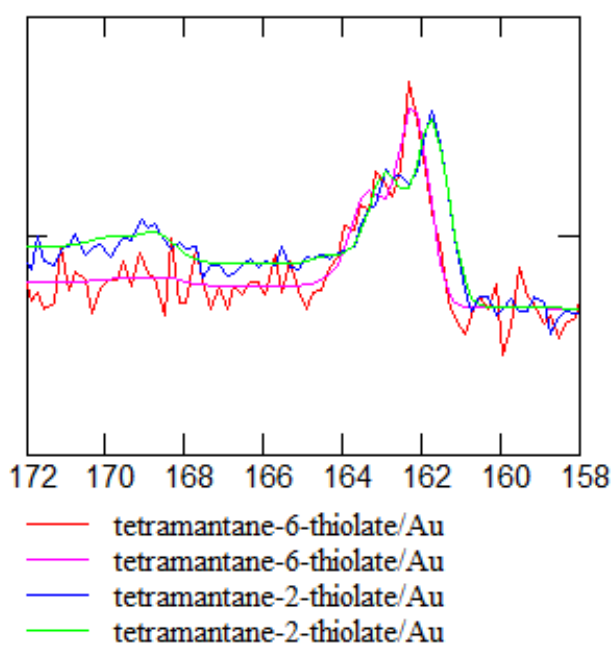

In this case, tetramantane-6-thiolate on gold was a clean monolayer, with only bound thiolate. The tetramantane-2-thiolate required multiple chemical components to fit the spectra, including significant elemental sulfur and oxidized sulfur species. The layer is good, but not pristine. The elemental sulfur makes the thiolate componenent difficult to deconvolute in this case, leading to larger errors in the binding energy. The tetramantane-2-thiol appears to have lower thiolate $S 2 p$ core-level binding energy than tetramantane-6-thiol. 
Additional data on the tetramantanethiols:
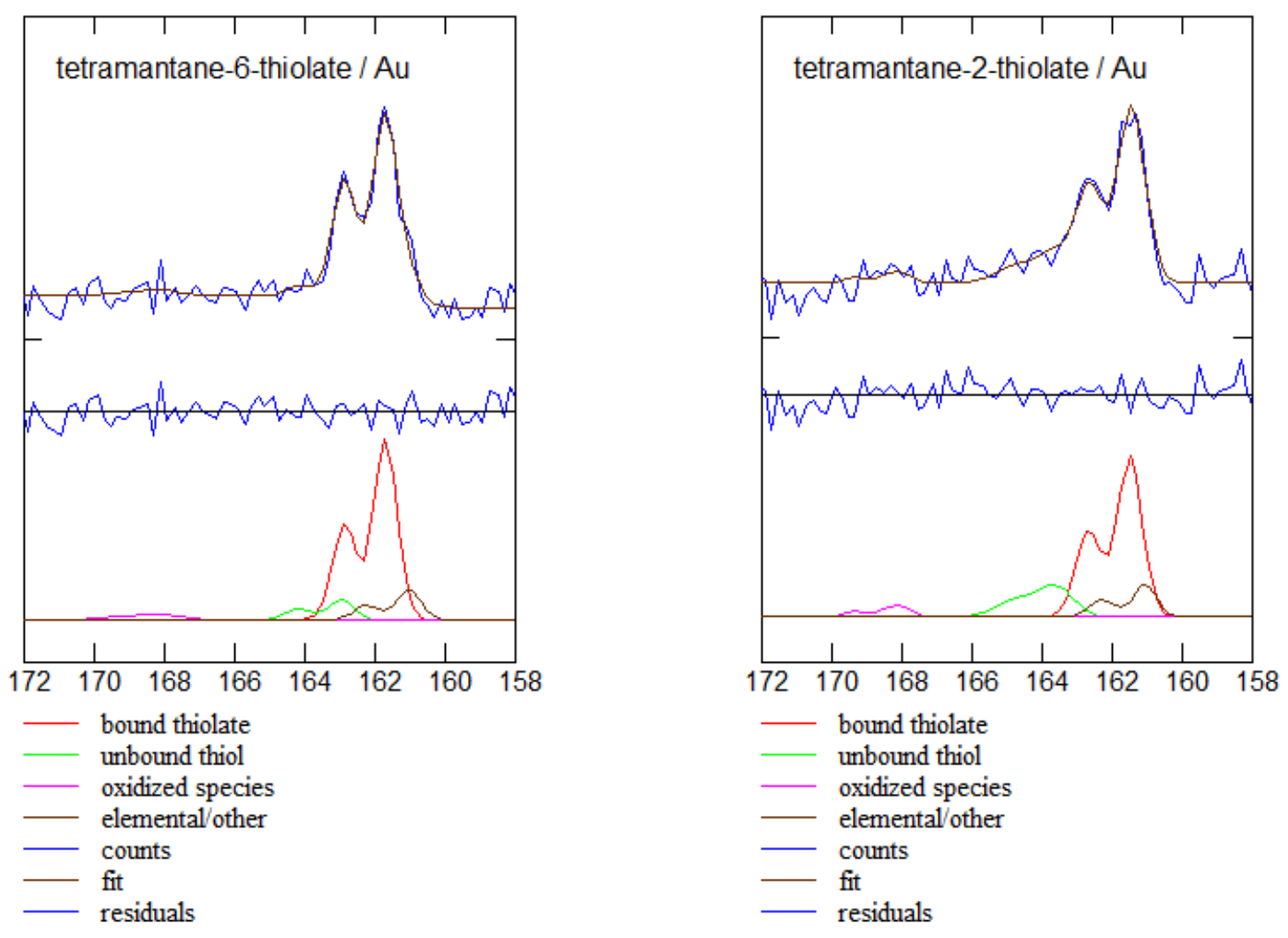

tetramantanethiolates / $\mathrm{Au}$

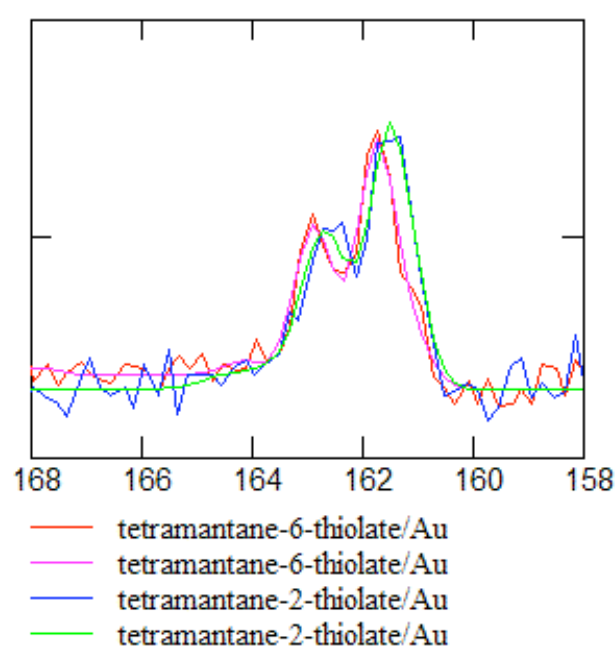

In this case, tetramantane-6-thiolate on gold had a small shoulder to lower binding energy (presumably elemental sulfur) while tetramantane-2-thiolate also had contaminant sulfur. In both cases, the tetramantane-2-thiolate/Au component appears to be slightly lower in binding energy. These data, along with additional S $2 p$ spectra acquired on tetramantanethiols (not shown) led to an estimate that tetramantane-6-thiolate on Au has a binding energy that is lower than dodecanethiolate on Au by $0.18+/$ $0.07 \mathrm{eV}$. For tetramantane-2-thiolate on $\mathrm{Au}$, we estimate that the binding energy is lower than dodecanethiolate by $0.49+/-0.18 \mathrm{eV}$. 
$\mathrm{S} 2 \mathrm{p}$ data on the triamantanethiols:
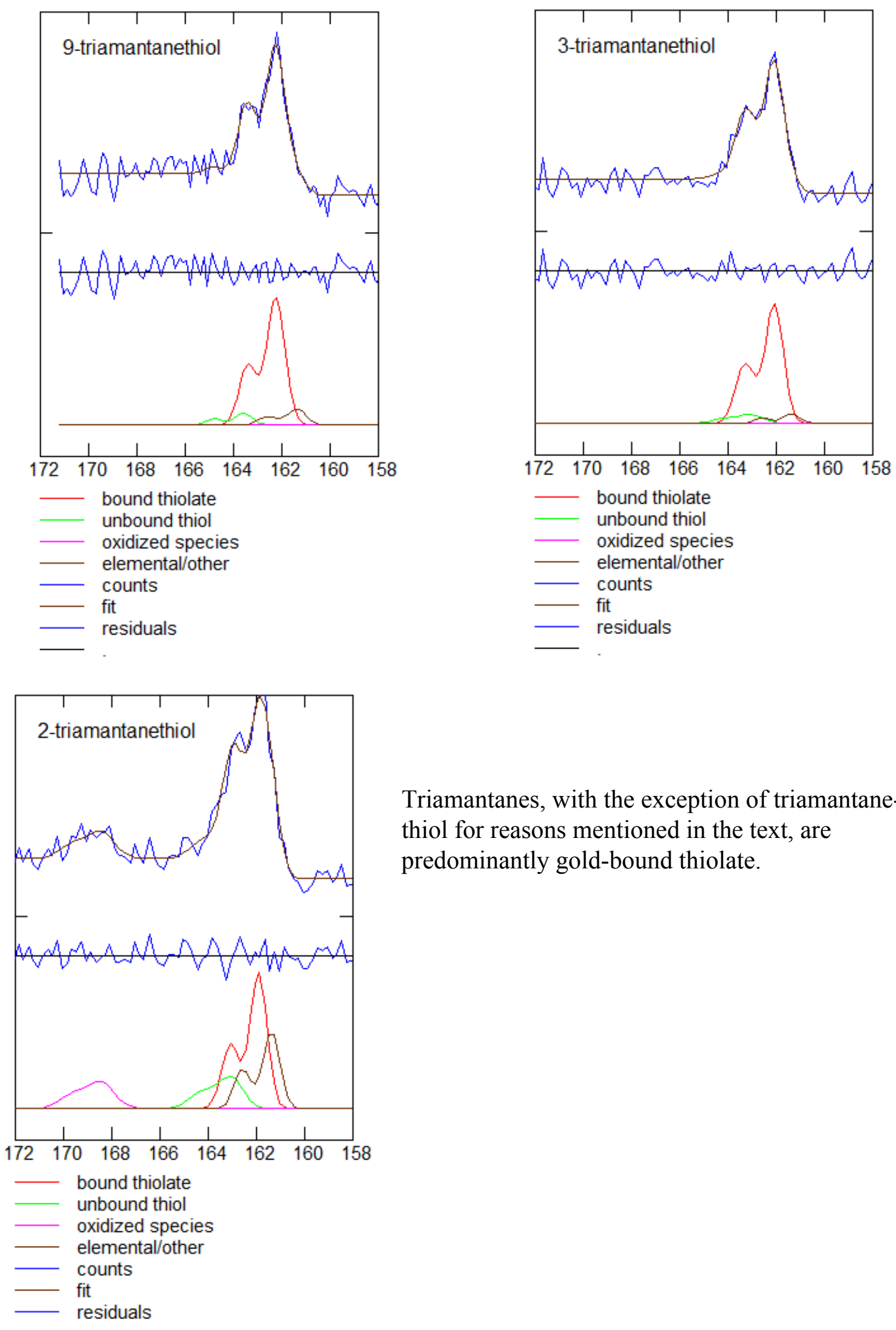

Triamantanes, with the exception of triamantane-2thiol for reasons mentioned in the text, are predominantly gold-bound thiolate. 
S $2 p$ data on the diamantanethiols:
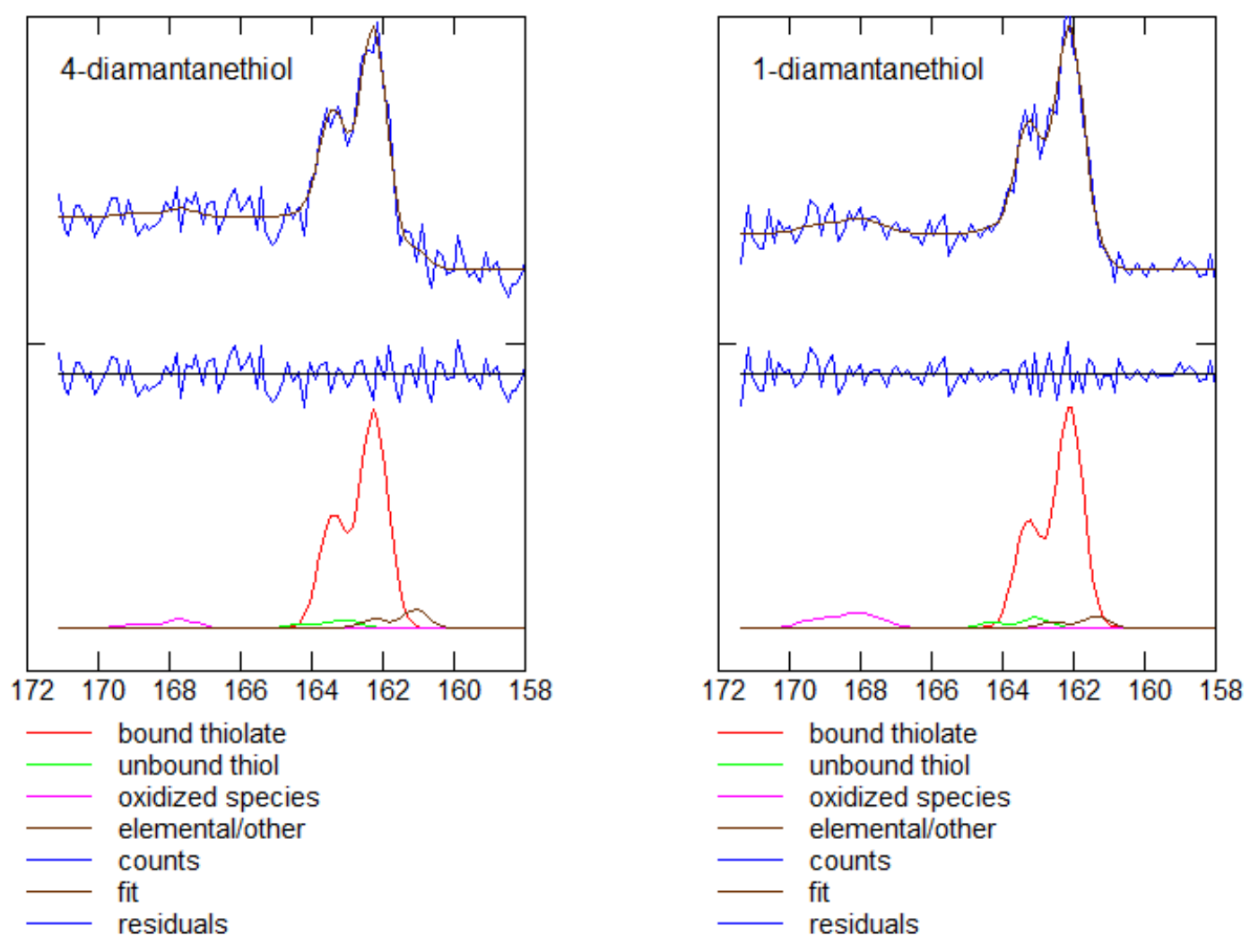

Both diamantanes exhibit clean gold-bound thiolate. 


\section{Additional Experimental Details:}

The $\mathrm{Au}(111)$ substrates were prepared under high vacuum by the thermal evaporative deposition of $5 \mathrm{~nm}$ of Ti followed by $500-1000 \mathrm{~nm}$ of Au onto native oxide $\mathrm{Si}(100)$ wafers. Prior to immersion in a diamondoid thiol solution, each substrate was gently annealed with a hydrogen flame to give reasonably large $\mathrm{Au}(111)$ domains at the surface[S2]. After removal from the diamondoid thiol solutions, samples were rinsed with clean ethanol, carefully dried with nitrogen, and quickly loaded into the vacuum chamber and pumped to ultra-high vacuum (UHV) below $\sim 10^{-9}$ torr to minimize exposure to ambient laboratory conditions.

X-ray absorption and X-ray photoemission spectra were recorded on beamlines (BL) 8.2 and 10.1 of the Stanford Synchrotron Radiation Laboratory (SSRL, SPEARIII) at the Stanford Linear Accelerator (SLAC). BL 8.2 is served by a bending magnet and a spherical grating monochromator and offers an energy resolution of about $0.1 \mathrm{eV}$ for NEXAFS experiments conducted at the carbon K-edge. BL 10.1 uses soft X-rays from a side branch of the beamline 10 wiggler and has a resolution of better than $0.05 \mathrm{eV}$. The cross-section of the focused beam was approximately $1 \mathrm{~mm}$ in diameter on both 8.2 and 10.1 at the sample surface. NEXAFS spectra were recorded simultaneously in both total electron yield (TEY) and Auger electron yield (AEY) modes. The TEY signal was obtained by measuring the total current leaving the experimental sample as the X-ray energy was scanned across the absorption edge. Measuring the intensity of the appropriate Auger electron during the course of each scan provided the AEY signal. The Auger electron intensity was recorded at a fixed kinetic energy and $200 \mathrm{eV}$ pass energy using a PHI15-255G cylindrical mirror analyzer (CMA) and associated OEM electronics. All NEXAFS signals were normalized to the $I_{0}$ current, which was recorded for the incident X-ray beam via a Au grid located upstream of the experimental sample. To ensure minimal effect on the $I_{0}$ signal from predominantly organic contaminants absorbed on the surface of the grid, it was frequently coated with a fresh layer of evaporated Au. All NEXAFS measurements were conducted at base pressures at or below about $1 \times 10^{-9}$ torr. The degree of linear polarization, $P$, in the incident beam was determined prior to each series of X-ray absorption experiments via carbon K-edge NEXAFS measurements of highly oriented pyrolytic graphite (HOPG). NEXAFS spectra were recorded at a series of angles of incidence between a freshly cleaved sample of HOPG and the X-ray beam. The axes through which the HOPG was rotated were carefully selected such that comparison of the $\mathrm{C}$ K-edge $\pi^{*}$ resonance intensity in the NEXAFS spectra yielded the relative magnitudes of $E_{p}{ }^{2}$ and $E_{s}{ }^{2}$, where $E_{p}$ and $E_{s}$ represent the electric field in-plane and perpendicular to the plane of incidence respectively[S3]. The calculated polarization was $99 \%$ in the plane of the storage ring for BL8.2, while the wiggler radiation from BL10.1 showed about $76 \%$ polarization during the course of the experiments presented in this paper. For carbon NEXAFS measurements, the energy scale was calibrated to the $\pi^{*}$ resonance for HOPG, for which the energy was assigned to be $285.38 \mathrm{eV}[\mathrm{S} 4]$. Care was taken to ensure that the effects of beam damage on the samples were minimized when conducting NEXAFS and PES measurements. Each spectrum was recorded from a fresh region of the sample surface and beam exposure during data collection was limited to the timeframe required for good signal to noise statistics. 
References:

S1: StoBe-deMon version 3.0 (2007), K. Hermann and L.G.M. Pettersson, M.E. Casida, C. Daul, A. Goursot, A. Koester, E. Proynov, A. St-Amant, and D.R. Salahub. Contributing authors: V. Carravetta, H. Duarte, C. Friedrich, N. Godbout, J. Guan, C. Jamorski, M. Leboeuf, M. Leetmaa, M. Nyberg, S. Patchkovskii, L. Pedocchi, F. Sim, L. Triguero, and A. Vela. See

S2: Molecular Imaging, Agilent Technologies. As of 4 December 2007, available online at: http://www.2spi.com/catalog/gold-sub2.html, or application note "hydrogen flame annealing" at

S3: Willey, T. M., Ph.D. Dissertation, University of California, Davis, 2004 (see Figure 2.7 and related text)

S4: Batson, P. E. Physical Review B 1993, 48, 2608-2610. 